\title{
Stability Switches and Hopf Bifurcation in a Coupled FitzHugh-Nagumo Neural System with Multiple Delays
}

\author{
Shengwei Yao ${ }^{1,2}$ and Huonian $\mathrm{Tu}^{2}$ \\ ${ }^{1}$ School of Science, East China University of Science Technology, Shanghai 200237, China \\ ${ }^{2}$ School of Information and Statistics, Guangxi University of Finance and Economics, Nanning 530003, China \\ Correspondence should be addressed to Shengwei Yao; idhot@163.com
}

Received 25 April 2014; Revised 10 June 2014; Accepted 11 June 2014; Published 8 July 2014

Academic Editor: Yongli Song

Copyright ( $\odot 2014$ S. Yao and H. Tu. This is an open access article distributed under the Creative Commons Attribution License, which permits unrestricted use, distribution, and reproduction in any medium, provided the original work is properly cited.

A FitzHugh-Nagumo (FHN) neural system with multiple delays has been proposed. The number of equilibrium point is analyzed. It implies that the neural system exhibits a unique equilibrium and three ones for the different values of coupling weight by employing the saddle-node bifurcation of nontrivial equilibrium point and transcritical bifurcation of trivial one. Further, the stability of equilibrium point is studied by analyzing the corresponding characteristic equation. Some stability criteria involving the multiple delays and coupling weight are obtained. The results show that the neural system exhibits the delay-independence and delay-dependence stability. Increasing delay induces the stability switching between resting state and periodic activity in some parameter regions of coupling weight. Finally, numerical simulations are taken to support the theoretical results.

\section{Introduction}

The FitzHugh-Nagumo (FHN) neuron $[1,2]$, a simplified model of Hodgkin-Huxley neuron [3], describes the generation and propagation of nerve impulse in planar autonomous systems. In recent years, to understand information processing in the brain, the FHN neuron model is commonly used to study neural spiking due to its simplicity. A complete qualitative investigation of the FHN neuron was done by Bautin [4]. Further, a hard oscillation, separatrix loops, and bifurcations of equilibria and limit cycles can occur under suitable values of the parameters in this system [5].

To understand the coupling effect and information transmission between neuron systems, the analysis of the dynamic behavior in coupled FHN neural systems has been the subject of many papers [6-9], in which many rich bifurcation behaviors for equilibrium point and limit cycle are observed. Based on the finite propagating speed in the signal transmission between the neurons [10, 11], a coupled FHN neural system with time delays has been established:

$$
\begin{aligned}
& \dot{u}_{1}=-u_{1}\left(u_{1}-1\right)\left(u_{1}-a\right)-u_{2}+c \tanh \left(u_{3}\left(t-\tau_{1}\right)\right), \\
& \dot{u}_{2}=b\left(u_{1}-\gamma u_{2}\right),
\end{aligned}
$$

$$
\begin{aligned}
& \dot{u}_{3}=-u_{3}\left(u_{3}-1\right)\left(u_{3}-a\right)-u_{4}+c \tanh \left(u_{1}\left(t-\tau_{2}\right)\right), \\
& \dot{u}_{4}=b\left(u_{3}-\gamma u_{4}\right)
\end{aligned}
$$

where $a, b$, and $\gamma$ are the positive constants, $u_{1,3}$ represent the membrane potentials of neurons 1 and $2, u_{2,4}$ are the slow refractory variables, which model the time dependence of several physical quantities, $c$ measures the coupling weight, and $\tau_{1,2}>0$ represent the time delays in signal transmission between the neurons.

The research for coupled FHN neural systems with time delay has attracted many authors' attentions. Burić and Todorović [12] studied the Hopf bifurcation (inverse and direct) and fold bifurcation of limit cycle in the delaycoupling FHN neurons. Using the ODE (ordinary differential equation) to approximate the dynamics of the coupled FHN neurons with small delays, the codimension-2 generalized Hopf bifurcation was investigated [13]. The different synchronization states were observed with the variation of the coupling strength and time delay [14]. Their results illustrated that the patterns of exactly synchronous oscillations were dependent on the type of excitability and coupling. 
Wang et al. [15] presented that time delay can control the transition from the original chaotic motions to periodic ones in two coupled nonidentical FHN models with synaptic connection. Regarding the sum of two delays as a parameter, Fan and Hong [16] investigated the stability and local Hopf bifurcation in the synaptically coupled nonidentical FHN model. The simple zero singularity (both pitchfork and transcritical bifurcations) was studied employing the center manifold reduction and normal form method [17]. Further, by applying the Bautin bifurcation theorem, the coexistence of rest state and periodic spiking was reported in the synchronous solution of a coupled FHN neural system with delay [18].

Recently, some criteria to determine the periodic oscillation were provided in the multiple delayed FHN neural system with three nonidentical cells [19]. In [20], the Hopf bifurcation and Bogdanov-Takens bifurcation were investigated in a coupled FHN neural system with gap junction. The spatiotemporal patterns of bifurcating periodic solutions were considered by using the symmetric bifurcation theory. The neuron behavior can transit between resting and spiking. By the proportional-spatial derivative control approach, Yang et al. [21] achieved the control problem of the FHN equation. All works mentioned above have promoted greatly a deep understanding for dynamic behavior of the coupled FHN systems with time delay. However, in reality, the neural dynamics may be influenced by multiple independent parameters, such as external inputs $[22,23]$, time delays, and coupling weight $[24,25]$. Their combined effects on dynamic behavior may be an important topic in the coupled FHN neural systems with multiple delays. The purpose of the present paper is to consider the combined effects of coupling weight and multiple delays on the stability of equilibrium point and obtain the stability switches in the coupled FHN neural system with multiple delays (1).

The paper is organized as follows. In the next section, we study the number of equilibrium points in the coupled FHN neural system employing the static bifurcation. The neural system (1) may have one/three equilibrium points for the varying of the coupled weight by employing the saddle-node bifurcation of nontrivial equilibrium point and transcritical bifurcation of trivial one. In Section 3, the stability of equilibrium point for system (1) is investigated by analyzing the corresponding characteristic equation. Some stability criteria involving the multiple delays and coupling weight are obtained. The results show that the FHN neural system exhibits the parameter regions involving the delay-independence stability and delay-dependence stability. Increasing time delay can induce the stability switches between resting state and periodic activity in some parameter regions of coupling weight. In Section 4, some numerical simulations are employed to support the theoretical results. Conclusions are given in Section 5.

\section{Equilibrium Point Analysis}

It is obvious that $\left(u_{1}, u_{2}, u_{3}, u_{4}\right)=(0,0,0,0)$ is the trivial equilibrium point of system (1). Linearizing system (1) near the point $(0,0,0,0)$ produces the following linearized system:

$$
\begin{aligned}
& \dot{u}_{1}=-a u_{1}-u_{2}+c u_{3}\left(t-\tau_{1}\right), \\
& \dot{u}_{2}=b u_{1}-b \gamma u_{2}, \\
& \dot{u}_{3}=-a u_{3}-u_{4}+c u_{1}\left(t-\tau_{2}\right), \\
& \dot{u}_{4}=b u_{3}-b \gamma u_{4} .
\end{aligned}
$$

The corresponding characteristic equation is

$$
D\left(\lambda, \tau_{1}, \tau_{2}, c\right)=\lambda^{4}+d_{1} \lambda^{3}+d_{2} \lambda^{2}+d_{3} \lambda+d_{4}=0,
$$

where

$$
\begin{aligned}
& d_{1}=2 a+2 b \gamma, \\
& d_{2}=a^{2}+2 b-c^{2} \mathrm{e}^{-\lambda \tau_{1}-\lambda \tau_{2}}+4 a b \gamma+b^{2} \gamma^{2}, \\
& d_{3}=2 a b+2 a^{2} b \gamma+2 b^{2} \gamma-2 b c^{2} \mathrm{e}^{-\lambda \tau_{1}-\lambda \tau_{2}} \gamma+2 a b^{2} \gamma^{2}, \\
& d_{4}=b^{2}+2 a b^{2} \gamma+a^{2} b^{2} \gamma^{2}-b^{2} c^{2} \mathrm{e}^{-\lambda \tau_{1}-\lambda \tau_{2}} \gamma^{2} .
\end{aligned}
$$

Then, we have the following conclusion on the eigenvalues of system (1).

Theorem 1. System (1) has a zero eigenvalue if and only if

$$
c= \pm \frac{1+a \gamma}{\gamma}, \quad \tau_{1} \neq \frac{2-b \gamma\left(\tau_{2}+\gamma\left(2+a \tau_{2}\right)\right)}{b \gamma(1+a \gamma)} .
$$

Further, system (1) exhibits a double zero eigenvalue if and only if

$$
\begin{aligned}
& c= \pm \frac{1+a \gamma}{\gamma}, \quad \tau_{1}=\frac{2-b \gamma\left(\tau_{2}+\gamma\left(2+a \tau_{2}\right)\right)}{b \gamma(1+a \gamma)}, \\
& a \neq \frac{-1-2 b \gamma^{2}+b^{2} \gamma^{4}}{2 \gamma} .
\end{aligned}
$$

Proof. $\lambda=0$ is a zero eigenvalue of system (1) if and only if

$$
\begin{gathered}
b^{2}+2 a b^{2} \gamma+a^{2} b^{2} \gamma^{2}-b^{2} c^{2} \gamma^{2}=0, \\
\left.\frac{\partial D(\lambda, c)}{\partial \lambda}\right|_{\lambda=0}=2 a b+2 a^{2} b \gamma+2 b^{2} \gamma-2 b c^{2} \gamma \\
+2 a b^{2} \gamma^{2}+b^{2} c^{2} \gamma^{2}\left(\tau_{1}+\tau_{2}\right) \neq 0 .
\end{gathered}
$$

Further, $\lambda=0$ is a double root of system (1) if and only if

$$
\begin{gathered}
c= \pm \frac{1+a \gamma}{\gamma}, \quad \tau_{1}=\frac{2-b \gamma\left(\tau_{2}+\gamma\left(2+a \tau_{2}\right)\right)}{b \gamma(1+a \gamma)}, \\
\left.\frac{\partial^{2} D(\lambda, c)}{\partial \lambda^{2}}\right|_{\lambda=0}=\frac{2+4 a \gamma+4 b \gamma^{2}-2 b^{2} \gamma^{4}}{\gamma^{2}} \neq 0 .
\end{gathered}
$$




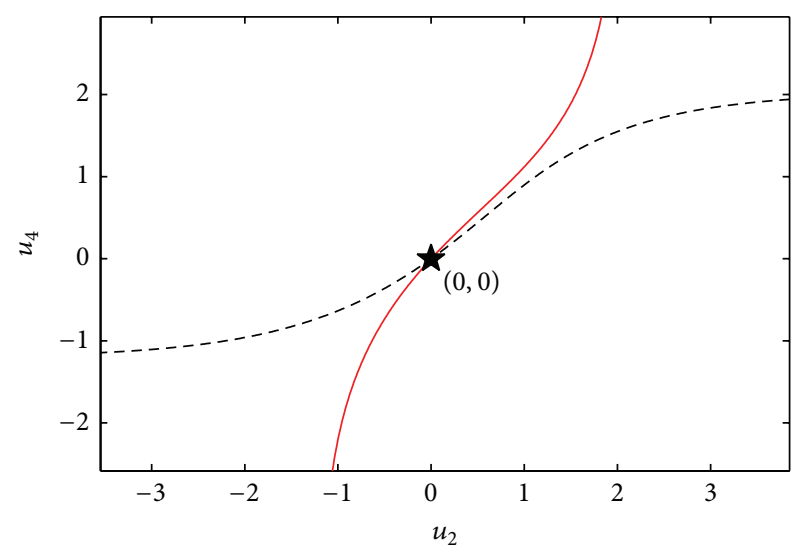

(a)

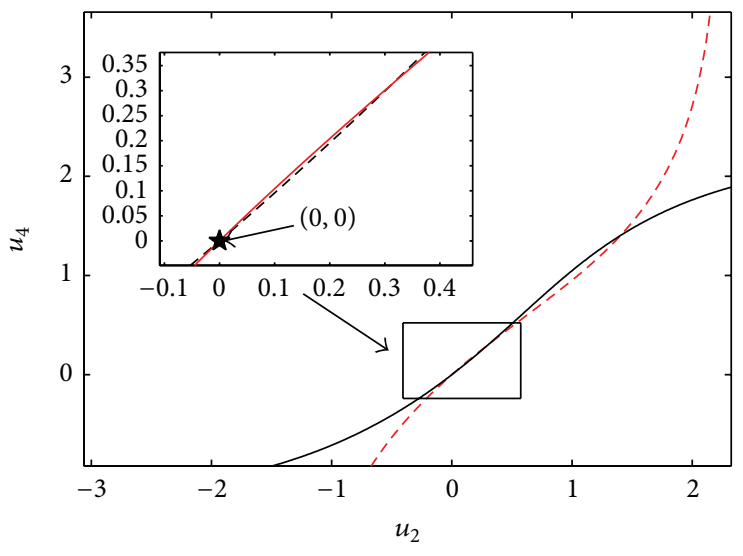

(b)

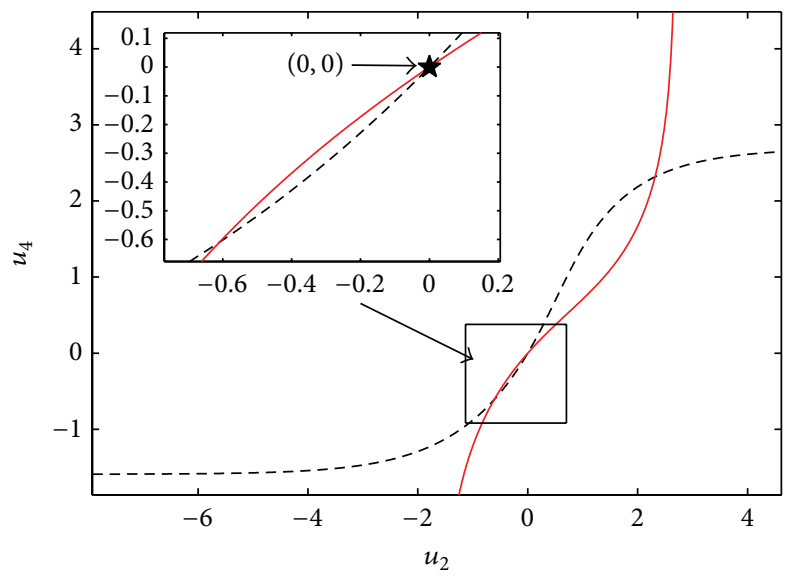

(c)

FIGURE 1: Intersection points of two curves given by (9), showing the number of equilibriums: (a) unique equilibrium for $c=2.0$, (b) three equilibriums for $c=2.3$, and (c) three equilibriums for $c=3.0$, where the system parameter values are fixed as $a=0.33, b=1$, and $\gamma=0.47$.

From the dynamic theory, when an eigenvalue passes through the imaginary axis along the real axis with the variation of system parameter, a static bifurcation will be exhibited, which results in the variation of the number of equilibrium points. In fact, all equilibrium points of system (1) are the solution of the following equations:

$$
\begin{aligned}
& \gamma u_{2}\left(\gamma u_{2}-1\right)\left(\gamma u_{2}-a\right)+u_{2}-c \tanh \left(\gamma u_{4}\right)=0, \\
& \gamma u_{4}\left(\gamma u_{4}-1\right)\left(\gamma u_{4}-a\right)+u_{4}-c \tanh \left(\gamma u_{2}\right)=0 .
\end{aligned}
$$

Solutions of (9), that is, equilibrium points of system (1), depend on the parameters $a, c$, and $\gamma$ but are independent of the delays $\tau_{1}$ and $\tau_{2}$. Geometrically, equilibrium points are the intersection points of two curves given by (9), as shown in Figure 1. These two curves are called the nullclines of system (1). Thus, it is seen that system (1) exhibits either one or three equilibriums for the different coupling weight $c$. In fact, for the small value of the coupling weight $c$, the system has just the trivial equilibrium point $(0,0)$, as shown in Figure 1(a). With the increasing of the coupling weight $c$, two nontrivial equilibrium points are emerged immediately at the right side of the trivial point $(0,0)$ (Figure $1(\mathrm{~b})$ ), which is the saddlenode bifurcation of the nontrivial equilibrium point. Further, the trivial equilibrium point $(0,0)$ evolves into somewhere between the nontrivial ones, as shown in Figure 1(c). It is called the transcritical bifurcation of the trivial point. Figure 2 shows the corresponding one-dimension bifurcation diagram in the $\left(c, u_{1}\right)$-plane (Figure $\left.2(\mathrm{a})\right)$ and $\left(c, u_{2}\right)$-plane (Figure 2(b)).

\section{Stability Switches and Hopf Bifurcation}

It is well known that the equilibrium point is locally asymptotically stable if and only if each eigenvalue of the characteristic equation (3) has the negative real part. In the following, we investigate the stability of the equilibrium point by obtaining the eigenvalues with the maximum real part. The FHN neural system exhibits the parameter regions involving the delay-independence stability and delay-dependence stability. The multiple delays can induce the stability switches between resting state and periodic activity in some parameter regions of coupling weight. To simplify, the investigation begins with the case $\tau_{1}=\tau_{2}=0$ in (3) as follows:

$$
D(\lambda, 0,0, c)=\lambda^{4}+m_{3} \lambda^{3}+m_{2} \lambda^{2}+m_{1} \lambda+m_{0}=0,
$$




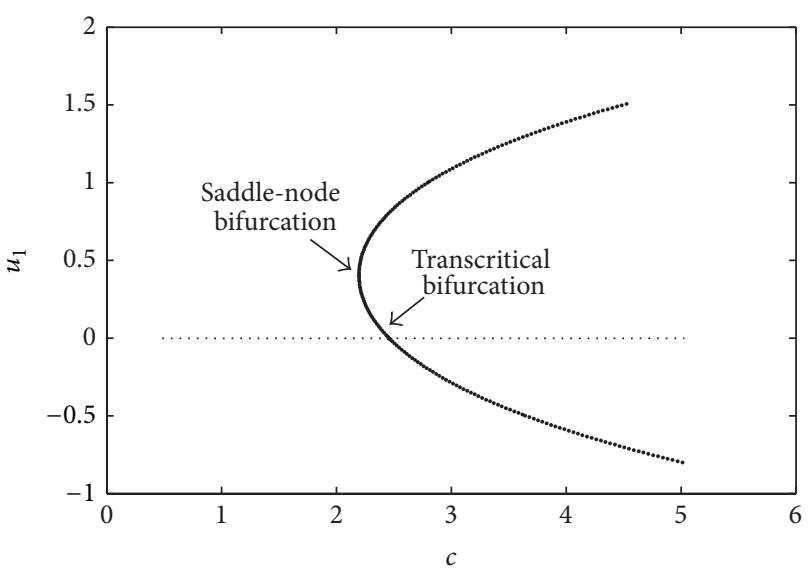

(a)

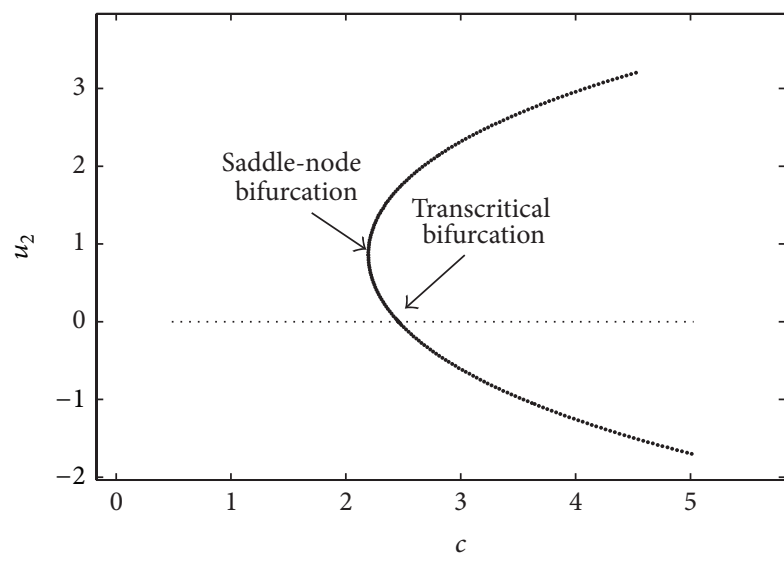

(b)

FIgURE 2: The one-dimensional bifurcation diagrams in the (a) $\left(c, u_{1}\right)$-plane and (b) $\left(c, u_{2}\right)$-plane for the fixed parameters $a=0.33, b=1$, and $\gamma=0.47$.

where

$$
\begin{aligned}
& m_{3}=2 a+2 b \gamma, \\
& m_{2}=a^{2}+2 b-c^{2}+4 a b \gamma+b^{2} \gamma^{2}, \\
& m_{1}=2 a b+2 a^{2} b \gamma+2 b^{2} \gamma-2 b c^{2} \gamma+2 a b^{2} \gamma^{2}, \\
& m_{0}=b^{2}+2 a b^{2} \gamma+a^{2} b^{2} \gamma^{2}-b^{2} c^{2} \gamma^{2} .
\end{aligned}
$$

It follows from the Routh-Hurwitz criterion that the necessary and sufficient conditions for all roots of (10) having negative real parts are given by

$$
\begin{aligned}
& m_{3}>0, \quad m_{0}>0, \quad m_{2} m_{3}-m_{1}>0, \\
& m_{1} m_{2} m_{3}-m_{1}^{2}-m_{0} m_{3}^{2}>0 .
\end{aligned}
$$

That is

(i) $1+a \gamma>c \gamma$ and $1+b \gamma>c$,

or

(ii) $1+a \gamma>c \gamma, a^{2}+a^{3} \gamma+2 a b \gamma+2 a^{2} b \gamma+b^{2} \gamma^{2}+a b^{2} \gamma^{3}<$ $a c^{2} \gamma$, and $a^{3}+a b+4 a^{2} b \gamma+b^{2} \gamma^{2}+4 a b^{2} \gamma^{3}+b^{3} \gamma^{3}>a c^{2}$.

Based on the dynamical theory, we have the following.

Theorem 2. If the system parameters are satisfied with one of the conditions ( $i$ ) and (ii), the trivial equilibrium point is locally asymptotically stable for the FHN neural system model without any time delays.

With the variation of delay $\tau_{1}$, the trivial equilibrium point of system (1) will lose its stability. The neural system may exhibit the periodic activity. To obtain such critical values, supposing $\lambda= \pm i v(v>0)$ is a pair of purely imaginary roots of the characteristic equation (3) with the case $\tau_{2}=0$, one has

$$
D\left(i v, \tau_{1}, 0, c\right)=v^{4}+d_{3} v^{3}+d_{2} v^{2}+d_{1} v+d_{0}=0,
$$

where

$$
\begin{aligned}
& d_{3}=-2 i a-2 i b \gamma \\
& d_{2}=c^{2} \mathrm{e}^{-i v \tau_{1}}-a^{2}-2 b-4 a b \gamma-b^{2} \gamma^{2}, \\
& d_{1}=2 i a b-2 i b c^{2} \gamma \mathrm{e}^{-i v \tau_{1}}+2 i a^{2} b \gamma+2 i b^{2} \gamma+2 i a b^{2} \gamma^{2}, \\
& d_{0}=b^{2}+2 a b^{2} \gamma-b^{2} c^{2} \gamma^{2} \mathrm{e}^{-i v \tau_{1}}+a^{2} b^{2} \gamma^{2} .
\end{aligned}
$$

That is

$$
\begin{aligned}
v^{4} & -2 i(a+b \gamma) v^{3}-\left(4 a b \gamma+b^{2} \gamma^{2}+a^{2}+2 b\right) v^{2} \\
& +2 i b\left(a+a^{2} \gamma+a b \gamma^{2}+b \gamma\right) v \\
& +\left(a^{2} \gamma^{2}+1+2 a \gamma\right) b^{2}+\left(v^{2}-2 i b v \gamma-b^{2} \gamma^{2}\right) c^{2} \cos v \tau_{1} \\
& -\left(i v^{2}+2 b v \gamma-i b^{2} \gamma^{2}\right) c^{2} \sin v \tau_{1}=0
\end{aligned}
$$

Separating (15) into the real and imaginary parts yields

$$
\begin{aligned}
& b^{2}-a^{2} v^{2}-2 b v^{2}+v^{4}+2 a b^{2} \gamma-4 a b v^{2} \gamma \\
& +a^{2} b^{2} \gamma^{2}-b^{2} v^{2} \gamma^{2}+\left(c^{2} v^{2}-b^{2} c^{2} \gamma^{2}\right) \cos v \tau_{1} \\
& \quad-2 b c^{2} v \gamma \sin v \tau_{1}=0, \\
& 2 a b v-2 a v^{3}+2 a^{2} b v \gamma+2 b^{2} v \gamma-2 b v^{3} \gamma+2 a b^{2} v \gamma^{2} \\
& \quad-2 b c^{2} v \gamma \cos v \tau_{1}+\left(b^{2} c^{2} \gamma^{2}-c^{2} v^{2}\right) \sin v \tau_{1}=0 .
\end{aligned}
$$

Eliminating $\tau_{1}$ from (16) using $\sin ^{2} v \tau_{1}+\cos ^{2} v \tau_{1}=1$, we have

$$
L(v)=v^{8}+n_{1} v^{6}+n_{2} v^{4}+n_{3} v^{2}+n_{4}=0,
$$


where

$$
\begin{aligned}
n_{1}= & 2 a^{2}-4 b+2 b^{2} \gamma^{2}, \\
n_{2}= & a^{4}-4 a^{2} b+6 b^{2}-c^{2}-c^{4}+4 a b^{2} \gamma \\
& +4 a^{2} b^{2} \gamma^{2}-4 b^{3} \gamma^{2}+b^{4} \gamma^{4}, \\
n_{3}= & 2 a^{2} b^{2}-4 b^{3}+4 a^{3} b^{2} \gamma-8 a b^{3} \gamma \\
& +2 a^{4} b^{2} \gamma^{2}-4 a^{2} b^{3} \gamma^{2}+2 b^{4} \gamma^{2} \\
& -2 b^{2} c^{2} \gamma^{2}-2 b^{2} c^{4} \gamma^{2}+4 a b^{4} \gamma^{3}+2 a^{2} b^{4} \gamma^{4}, \\
n_{4}= & b^{4}+4 a b^{4} \gamma+6 a^{2} b^{4} \gamma^{2}+4 a^{3} b^{4} \gamma^{3} \\
& +a^{4} b^{4} \gamma^{4}-b^{4} c^{2} \gamma^{4}-b^{4} c^{4} \gamma^{4} .
\end{aligned}
$$

Based on the dynamical theory, the equilibrium point is locally asymptotically stable if and only if each of eigenvalues has the negative real part. Therefore, one can obtain the effect of delay $\tau_{1}$ on the eigenvalue of characteristic equation (13).

Theorem 3. If the parameters values of system (1) are satisfied with one of the conditions (i) and (ii), the following statements are true.

(a) When the polynomial $L(v)$ has no positive root, all eigenvalues of the characteristic equation (13) have the negative real parts for the arbitrary $\tau_{1}$. The trivial equilibrium point exhibits the delay-independent stability.

(b) When the polynomial $L(v)$ has only one positive and simple root $v$, there exists the critical delayed value $\tau_{1}^{c}>0$ such that all the eigenvalues of the characteristic equation (13) have negative real parts for $\tau_{1} \in\left(0, \tau_{1}^{c}\right)$ and at least one root has a positive real part for $\tau_{1}>\tau_{1}^{c}$. The FHN neural system exhibits the periodic activity when delay $\tau_{1}$ increases and passes through the critical delayed value $\tau_{1}^{c}$.

(c) When the polynomial $L(v)$ has at least two positive and simple roots $0<v_{1}<v_{2}<\cdots$, there exist a finite number of the delayed $\tau_{1}$ intervals in which all eigenvalues of the characteristic equation (13) have negative real parts. The system exhibits the stability switches between resting state and periodic activity.

In order to investigate the combined effects of multiple delays on the local stability of neural system (1), we regard $\tau_{2}$ as the varying parameter for any fixed delay $\tau_{1}=\tau_{1}^{*}$. Letting $\lambda=i \omega$ be the simple roots of the characteristic equation (3), one obtains

$$
D\left(i \omega, \tau_{1}^{*}, \tau_{2}, c\right)=\omega^{4}+k_{3} \omega^{3}+k_{2} \omega^{2}+k_{1} \omega+k_{0}=0,
$$

where

$$
\begin{aligned}
& k_{3}=-2 i a-2 i b \gamma, \\
& k_{2}=-a^{2}-2 b+c^{2} \mathrm{e}^{-i \omega \tau_{1}^{*}} \mathrm{e}^{-i \omega \tau_{2}}-4 a b \gamma-b^{2} \gamma^{2},
\end{aligned}
$$

$$
\begin{aligned}
& k_{1}=2 i a b+2 i a^{2} b \gamma+2 i b^{2} \gamma-2 i b c^{2} \mathrm{e}^{-i \omega \tau_{1}^{*}} \mathrm{e}^{-i \omega \tau_{2}} \gamma+2 i a b^{2} \gamma^{2}, \\
& k_{0}=b^{2}+2 a b^{2} \gamma+a^{2} b^{2} \gamma^{2}-b^{2} c^{2} \mathrm{e}^{-i \omega \tau_{1}^{*}} \mathrm{e}^{-i \omega \tau_{2}} \gamma^{2} .
\end{aligned}
$$

That is

$$
\begin{aligned}
b^{2} & +2 a b^{2} \gamma+a^{2} b^{2} \gamma^{2}-\left(a^{2}+2 b+4 a b \gamma+b^{2} \gamma^{2}\right) \omega^{2} \\
& +\omega^{4}+\left(c^{2} \omega^{2}-b^{2} c^{2} \gamma^{2}\right) \cos \omega \tau_{2} \cos \omega \tau_{1}^{*} \\
& -2 b c^{2} \gamma \omega \cos \omega \tau_{1}^{*} \sin \omega \tau_{2}-2 b c^{2} \gamma \omega \cos \omega \tau_{2} \sin \omega \tau_{1}^{*} \\
& +\left(b^{2} c^{2} \gamma^{2}-c^{2} \omega^{2}\right) \sin \omega \tau_{2} \sin \omega \tau_{1}^{*}=0, \\
\left(2 a b+2 a^{2} b \gamma+2 b^{2} \gamma+2 a b^{2} \gamma^{2}\right) \omega-2(a+b \gamma) \omega^{3} & \\
& -2 b c^{2} \gamma \omega \cos \omega \tau_{2} \cos \omega \tau_{1}^{*} \\
& +\left(b^{2} c^{2} \gamma^{2}-c^{2} \omega^{2}\right) \cos \omega \tau_{1}^{*} \sin \omega \tau_{2} \\
& +\left(b^{2} c^{2} \gamma^{2}-c^{2} \omega^{2}\right) \cos \tau_{2} \omega \sin \omega \tau_{1}^{*} \\
& +2 b c^{2} \gamma \omega \sin \tau_{2} \omega \sin \omega \tau_{1}^{*}=0 .
\end{aligned}
$$

Eliminating $\tau_{2}$ from (21), one has

$$
\begin{aligned}
& \cos \omega \tau_{2}=\frac{f_{1}\left(\tau_{1}^{*}, \omega\right)}{f_{3}\left(\tau_{1}^{*}, \omega\right)}, \\
& \sin \omega \tau_{2}=\frac{f_{2}\left(\tau_{1}^{*}, \omega\right)}{f_{3}\left(\tau_{1}^{*}, \omega\right)},
\end{aligned}
$$

where

$$
\begin{gathered}
f_{1}\left(\tau_{1}^{*}, \omega\right) \\
=\left(b \omega+(a-\omega) \omega^{2}+b^{2} \gamma(1+a \gamma-\gamma \omega)\right) \\
\times\left(-b \omega+\omega^{2}(a+\omega)\right. \\
\left.+b^{2} \gamma(1+a \gamma+\omega \gamma)\right) \\
\times \cos \omega \tau_{1}^{*}-2 \omega\left(b\left(-1+b \gamma^{2}\right)+\omega^{2}\right) \\
\times\left(b^{2} \gamma(1+a \gamma)+a \omega^{2}\right) \sin \omega \tau_{1}^{*}, \\
f_{2}\left(\tau_{1}^{*}, \omega\right) \quad \\
=-\left(2 \omega\left(b\left(-1+b \gamma^{2}\right)+\omega^{2}\right)\right. \\
\times\left(b^{2} \gamma(1+a \gamma)+a \omega^{2}\right) \cos \omega \tau_{1}^{*} \\
+\left(b \omega+(a-\omega) \omega^{2}\right. \\
\left.\quad+b^{2} \gamma(1+a \gamma-\gamma \omega)\right) \\
\times\left(-b \omega+\omega^{2}(a+\omega)\right. \\
\left.\left.\quad+b^{2} \gamma(1+a \gamma+\omega \gamma)\right) \sin \omega \tau_{1}^{*}\right), \\
f_{3}\left(\tau_{1}^{*}, \omega\right)=c^{2}\left(b^{2} \gamma^{2}+\omega^{2}\right)^{2} .
\end{gathered}
$$




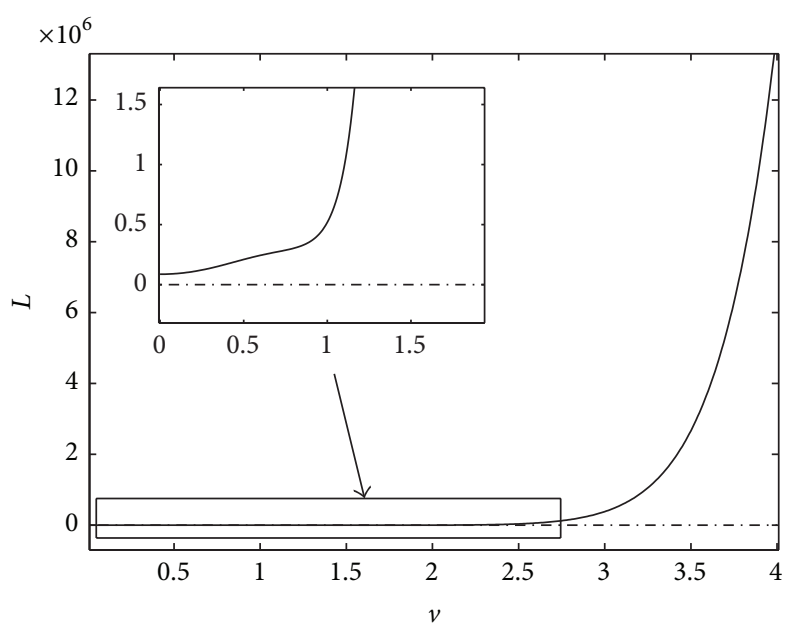

(a)

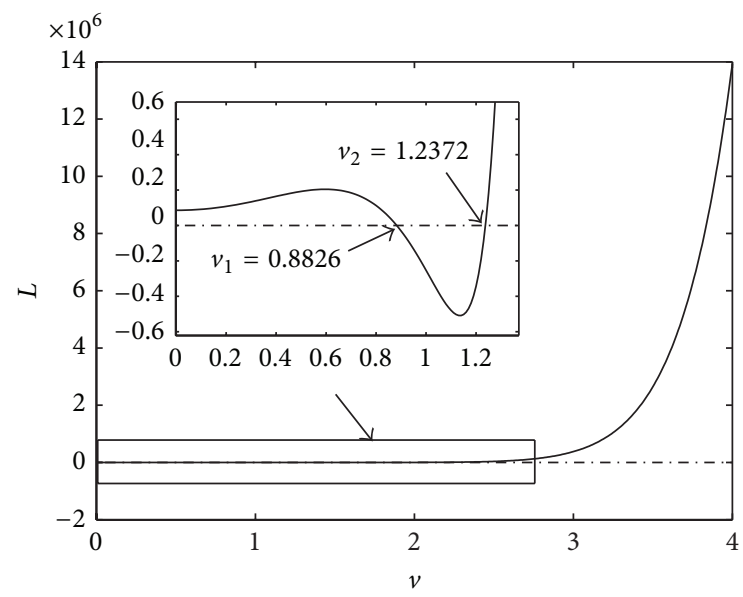

(c)

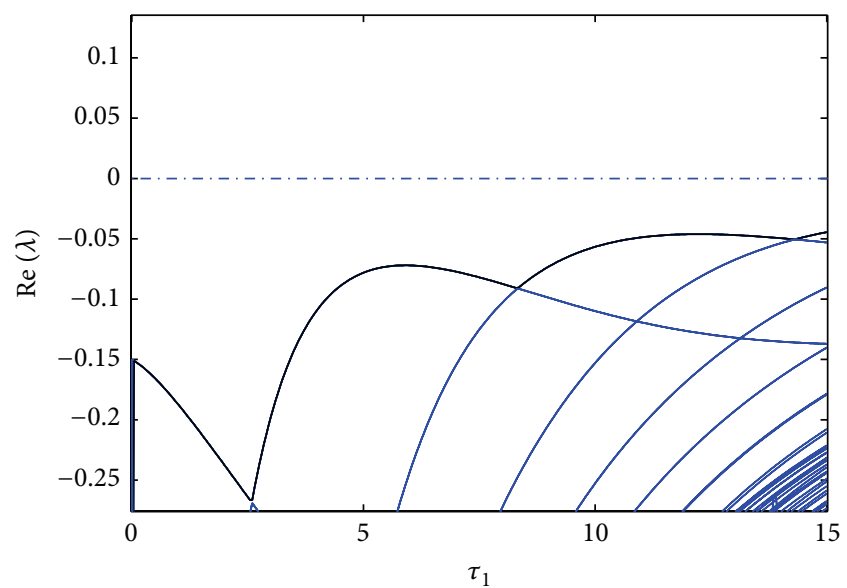

(b)

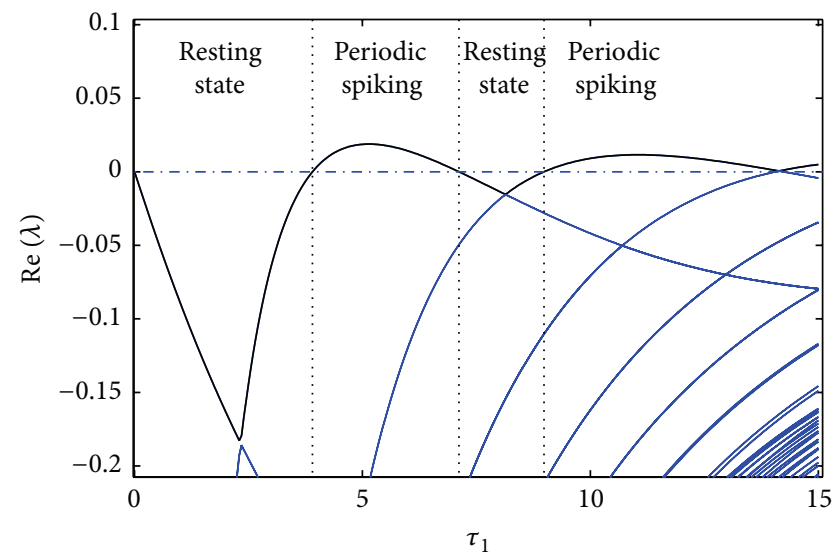

(d)

Figure 3: Roofs of function $L$ ((a) and (c)) and eigenvalue real parts with $\tau_{1}$ varying ((b) and (d)) for the different coupling weights (a)-(b) $c=0.5$ and $(\mathrm{c})-(\mathrm{d}) c=0.8$. The other parameters are chosen as $a=0.33, b=1, \gamma=0.47$, and $\tau_{2}=0$.

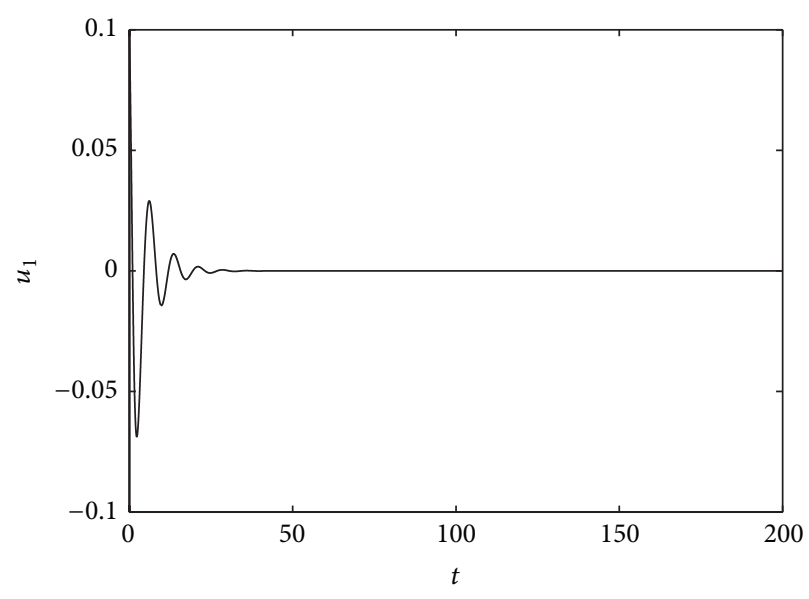

(a)

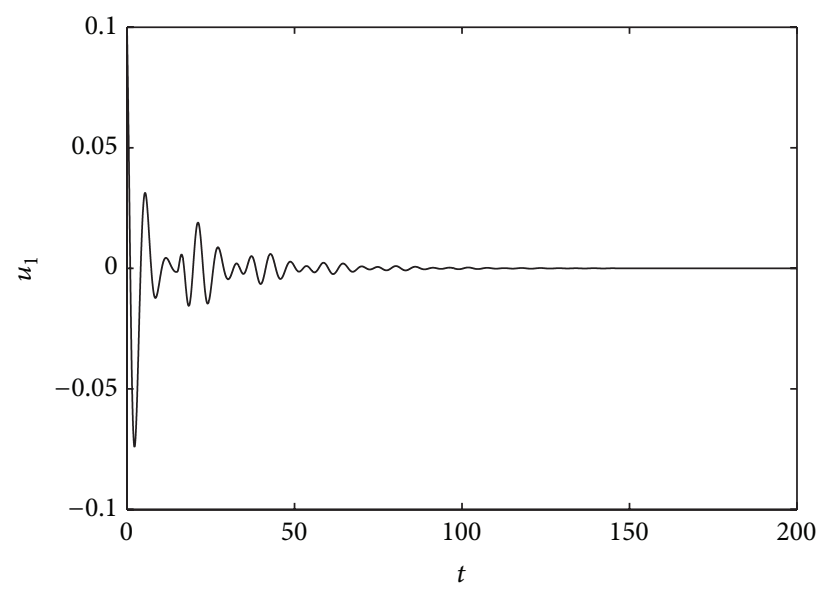

(b)

FIGURE 4: Time histories with the varying delay $\tau_{1}$ (a) $\tau_{1}=1.0$ and (b) $\tau_{1}=15.0$ when the time delay and coupling weight are fixed as $\tau_{2}=0$, $c=0.5$, where the other system parameters have the fixed values $a=0.33, b=1$, and $\gamma=0.47$. 


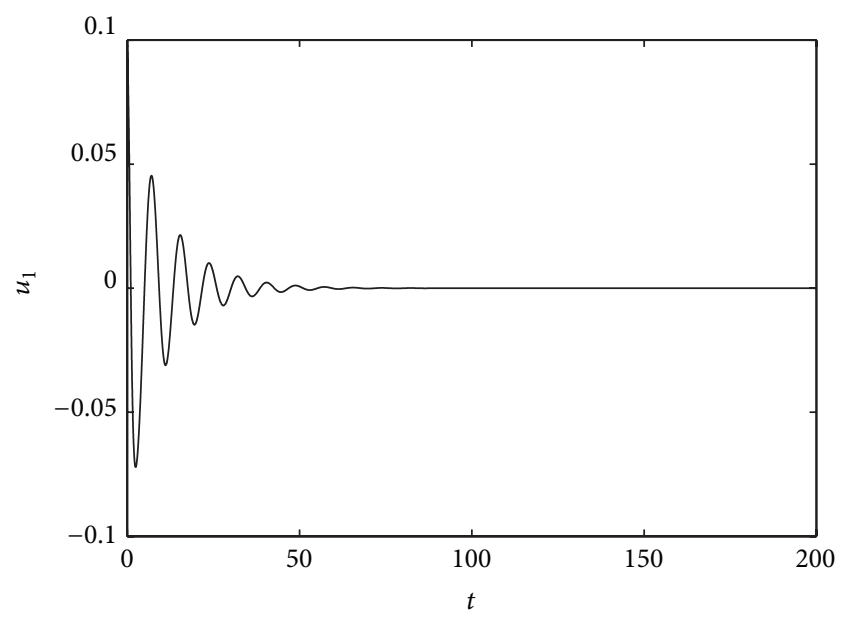

(a)

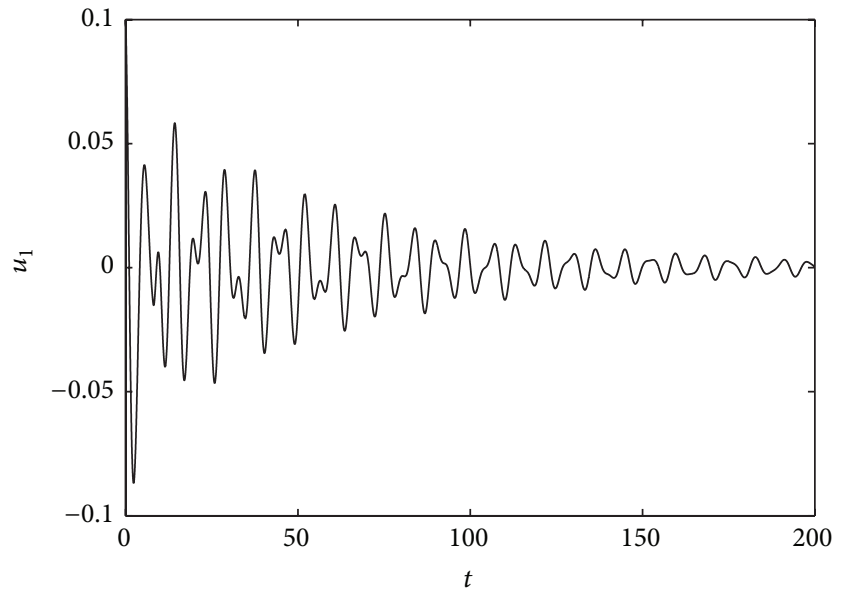

(c)

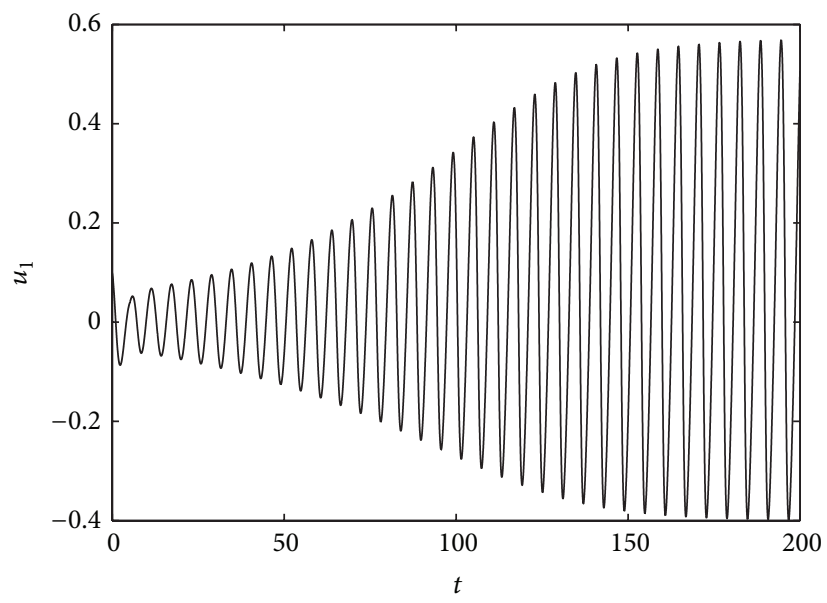

(b)

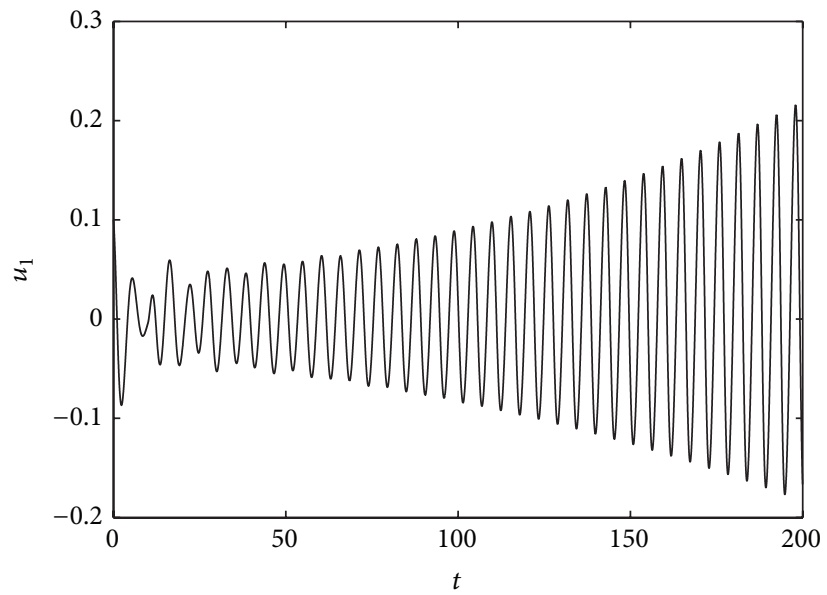

(d)

FIGURE 5: Time histories with the varying delay $\tau_{1}$ (a) $\tau_{1}=1.0$, (b) $\tau_{1}=5.0$, (c) $\tau_{1}=8.0$, and (d) $\tau_{1}=10.0$ when the delay $\tau_{2}$ and coupled weight are fixed as $\tau_{2}=0, c=0.8$, where the other system parameters have the fixed values $a=0.33, b=1$, and $\gamma=0.47$.

This implies that

$$
\begin{aligned}
G\left(\tau_{1}^{*}, \omega\right)= & \left(f_{1}\left(\tau_{1}^{*}, \omega\right)\right)^{2}+\left(f_{2}\left(\tau_{1}^{*}, \omega\right)\right)^{2} \\
& -\left(f_{3}\left(\tau_{1}^{*}, \omega\right)\right)^{2}=0 .
\end{aligned}
$$

If (24) has a number of positive and simple roots $\omega_{i}, i=$ $1,2, \ldots$, (3) has the following critical delays determined by (24):

$$
\tau_{i, j}=\frac{\left(\varphi_{i}+2 j \pi\right)}{\omega_{i}}, \quad i=1,2, \ldots ; j=0,1,2, \ldots,
$$

where $\varphi_{i} \in[0,2 \pi)$ and is satisfied with

$$
\cos \left(\varphi_{i}\right)=\frac{f_{1}\left(\tau_{1}^{*}, \omega_{i}\right)}{f_{3}\left(\tau_{1}^{*}, \omega_{i}\right)}, \quad \sin \left(\varphi_{i}\right)=\frac{f_{2}\left(\tau_{1}^{*}, \omega_{i}\right)}{f_{3}\left(\tau_{1}^{*}, \omega_{i}\right)}
$$

We determine whether the eigenvalue real part increases or decreases as delay $\tau_{2}$ crosses the critical values $\tau_{i, j}$, $i=1,2, \ldots ; j=0,1,2, \ldots$ Differentiating $\lambda$ with respect to $\tau_{2}$ in (3) reaches

$$
\lambda^{\prime}\left(\tau_{2}\right)=\frac{\lambda^{2} \mathrm{e}^{2 \lambda \tau_{1}^{*}} c^{2}(b \gamma+\lambda)^{2}}{g\left(\tau_{2}, \lambda\right)}
$$

where

$$
\begin{aligned}
g\left(\tau_{2}, \lambda\right)= & 2 \mathrm{e}^{\lambda\left(\tau_{1}^{*}+\tau_{2}\right)}(a+b \gamma+2 \lambda) \\
& \times(\lambda(a+\lambda)+b(1+a \gamma+\lambda \gamma)) \\
& +c^{2}(b \gamma+\lambda) \\
& \times\left(-2+(b \gamma+\lambda)\left(\tau_{1}^{*}+\tau_{2}\right)\right) .
\end{aligned}
$$

Based on the transversality condition (27) and the Hopf bifurcation theorem of delay differential equations, one has the following theorem. 


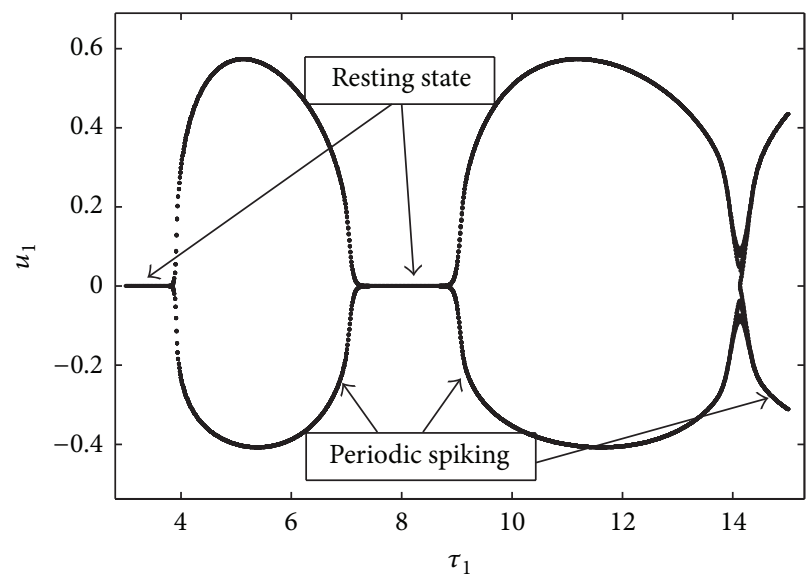

(a)

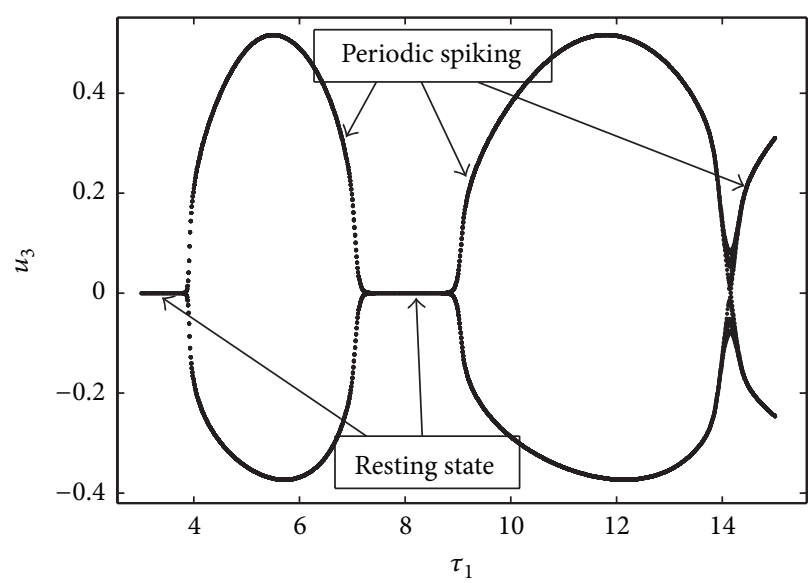

(c)

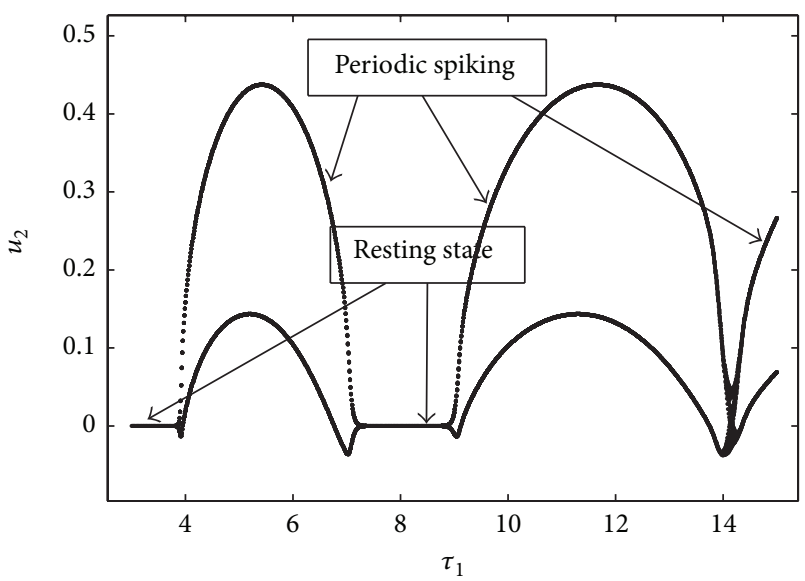

(b)

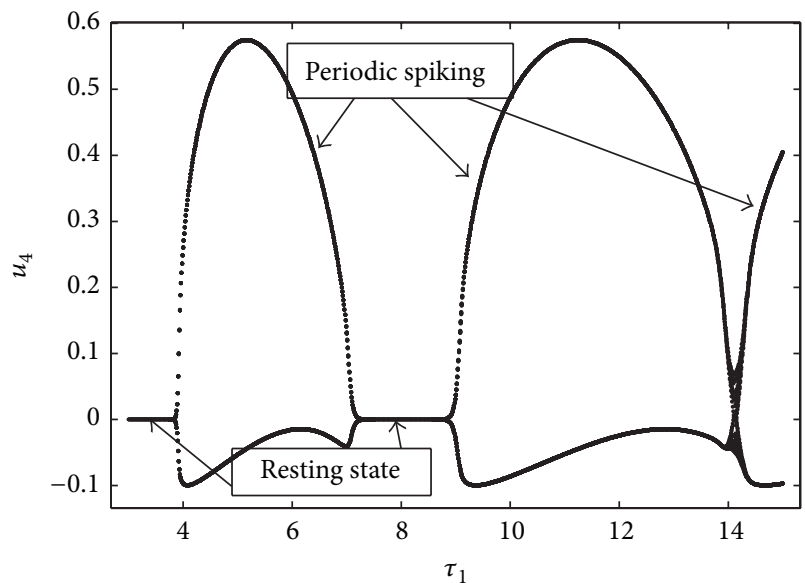

(d)

Figure 6: The one-dimensional bifurcation diagram in (a) $\left(\tau_{1}, u_{1}\right)$-plane, (b) $\left(\tau_{1}, u_{2}\right)$-plane, (c) $\left(\tau_{1}, u_{3}\right)$-plane, and (d) $\left(\tau_{1}, u_{4}\right)$-plane for the fixed delay $\tau_{2}=0$ and coupling weights $c=0.8$.

Theorem 4. The following assertions are true if all roots of (13) have negative real parts.

(a) If $G\left(\tau_{1}^{*}, \omega\right)=0$ has no positive root, the trivial equilibrium point of system (1) is asymptotically stable for an arbitrary time delay $\tau_{2}$, which is called the delay $\tau_{2}-$ independent stability.

(b) If $G\left(\tau_{1}^{*}, \omega\right)=0$ has at least one positive and simple root $\omega_{c}$, there exists exactly a critical delay $\tau_{2}^{c}>0$ such that the trivial equilibrium of system (1) is asymptotically stable for $\tau_{2} \in\left[0, \tau_{2}^{c}\right)$. Furthermore, if the transversality condition $\operatorname{Re}\left(\lambda^{\prime}\left(\tau_{2}\right)\right) \neq 0$ holds, system (1) undergoes a Hopf bifurcation for $\tau_{2}=\tau_{2}^{c}$. That is, it exhibits a periodic activity bifurcating from the trivial equilibrium near $\tau_{2}^{c}$.

(c) If $G\left(\tau_{1}^{*}, \omega\right)=0$ has at least two positive and simple roots $0<\omega_{1}<\omega_{2}<\cdots$ and the transversality condition $\operatorname{Re}\left(\lambda^{\prime}\left(\tau_{2}\right)\right) \neq 0$ holds, there exist a finite number of intervals. If time delay is fixed into these intervals, the trivial equilibrium point is locally asymptotically stable, while unstable if delay does not belong to ones. The rest sate of neural system switches from stable to unstable, and then back to stable when time delay increases and crosses the critical values in sequence.

\section{Numerical Simulation}

In this section, some numerical results of system (1) are presented for the different parameter values. Using the method of numerical simulation, we find that the theoretically predicted values are in excellent agreement with the numerical behaviors. For simplicity, the system parameters are fixed as $a=0.33, b=1$, and $\gamma=0.47$. The coupling weight $c$ and time delays $\left(\tau_{1}, \tau_{2}\right)$ are considered as the variable parameters.

Firstly, we fix the time delay $\tau_{2}=0$ and illustrate the dynamic behavior of system (1) for the different values of the coupling weight $c$ and time delay $\tau_{1}$. It follows from (17) that the polynomial $L(v)$ has no positive root (see Figure $3(\mathrm{a})$ ) for the fixed coupling weight $c=0.5$. The trivial equilibrium point is locally asymptotically stable for the arbitrary delay $\tau_{1}$, which is called the delay $\tau_{1}$-independent stability. The corresponding eigenvalue has the negative real parts for the delay $\tau_{1}$ variation, as shown in Figure $3(\mathrm{~b})$. The time histories 


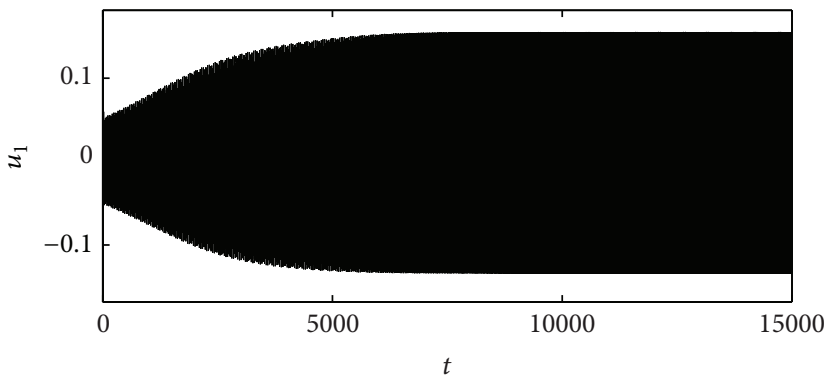

(a)

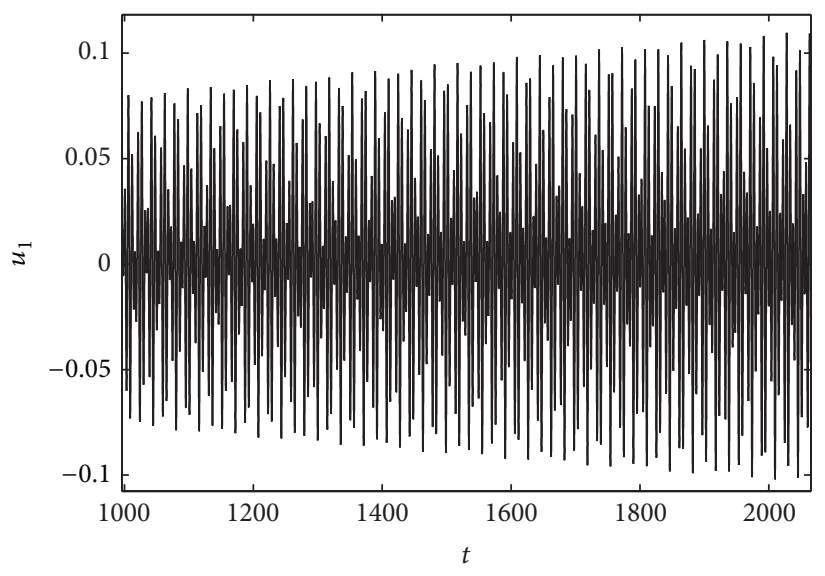

(b)

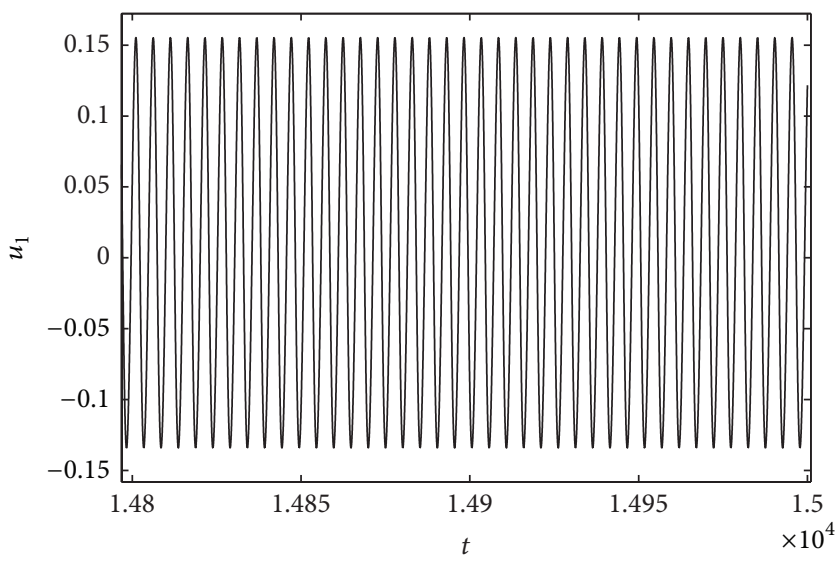

(c)

Figure 7: Time histories of the transient chaos for (a) overall view, (b) transient behavior, and (c) long-term behavior for the fixed delay $\tau_{1}=14.15$ when $\tau_{2}=0$ and $c=0.8$.

with the fixed delay are shown in Figure 4(a) for $\tau_{1}=1.0$ and Figure 4(b) for $\tau_{1}=15.0$; both converge to the equilibrium point $(0,0,0,0)$ spirally.

However, when the coupling weight is fixed as $c=0.8$, the equation $L(v)=0$ has two positive and simple roots $v_{1}=0.8826, v_{2}=1.2372$, as shown in Figure 3(c). The corresponding eigenvalue with $\tau_{1}$ varying is shown in Figure $3(\mathrm{~d})$. There exist some critical delays determined by (25). From Theorem 3, system (1) exhibits the stability switches with the delay $\tau_{1}$ increasing. In fact, the trivial equilibrium point is asymptotically stable when $\tau_{1} \in(0,3.9045)$. Figure 5(a) illustrates the time history for the fixed delay $\tau_{1}=1.0$. When $\tau_{1} \in(3.9045,7.1184)$, the trivial equilibrium point loses its stability and evolves into the instability. The time history for $\tau_{1}=5.0$ is shown in Figure 5(b). Further, system (1) obtains the resting state when delay increases and crosses the critical value $\tau_{1}=7.1184$. The time history for the fixed delay $\tau_{1}=$ 8.0 is illustrated in Figure 5(c). Finally, the equilibrium point loses its stability when the delay $\tau_{1}$ continues to increase, which is shown in Figure 5(d) for the fixed delay $\tau_{1}=10.0$. Figure 6 shows the corresponding one-dimension bifurcation diagrams for the varying delay $\tau_{1}$. It supports the above theoretical results. Neural system (1) exhibits the multiple switches between resting state and periodic spiking. Further, it is worth mentioning that the neural system (1) exhibits the transient chaos (see Figure $7(\mathrm{a})$ ), which implies that the system trajectory exhibits a seemingly chaotic solution for a longer time (see Figure 7(b)) but evolves into a final nonchaotic state, such as a periodic activity (see Figure 7(c)) or equilibrium eventually.

The partial eigenvalues are exhibited in Figure 8 for the different delay $\tau_{1}$ to illustrate the stability switches between resting state and periodic spiking. Figure 8 (a) shows that the maximum eigenvalues are a conjugate pair with negative part given by $-0.0888 \pm 0.7515 i$ for the fixed delay $\tau_{1}=1.0$. The neural system exhibits the resting state. Increasing delay $\tau_{1}$ yields that the conjugate pair passes through the imaginary axis and go into the right-half plane. For $\tau_{1}=5.0$, the pair becomes to be $0.0187 \pm 1.0846 i$ shown in Figure 8(b). The trivial equilibrium point loses its stability. The FHN neural system exhibits the periodic spiking. Continuing to increase delay $\tau_{1}$, the conjugate pair returns and passes through the imaginary axis again. The maximum eigenvalues with negative real parts occurs in the left-hand plane. 


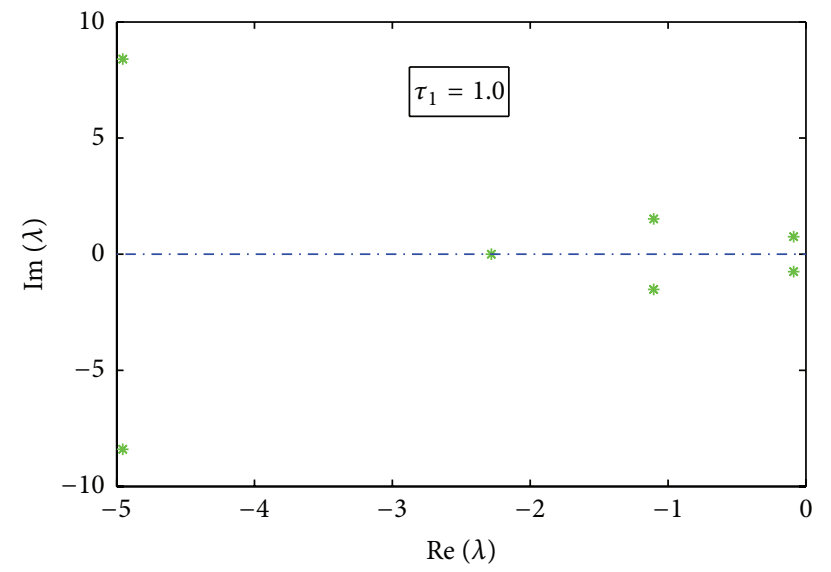

(a)

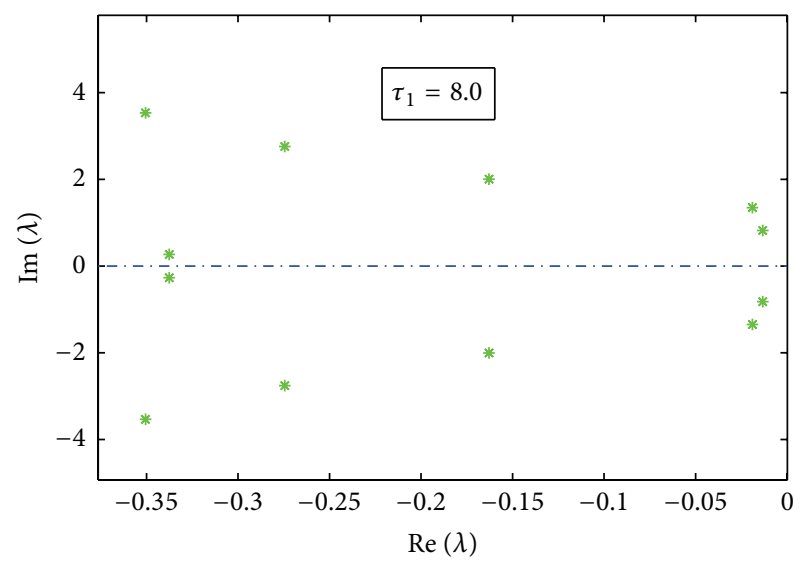

(c)

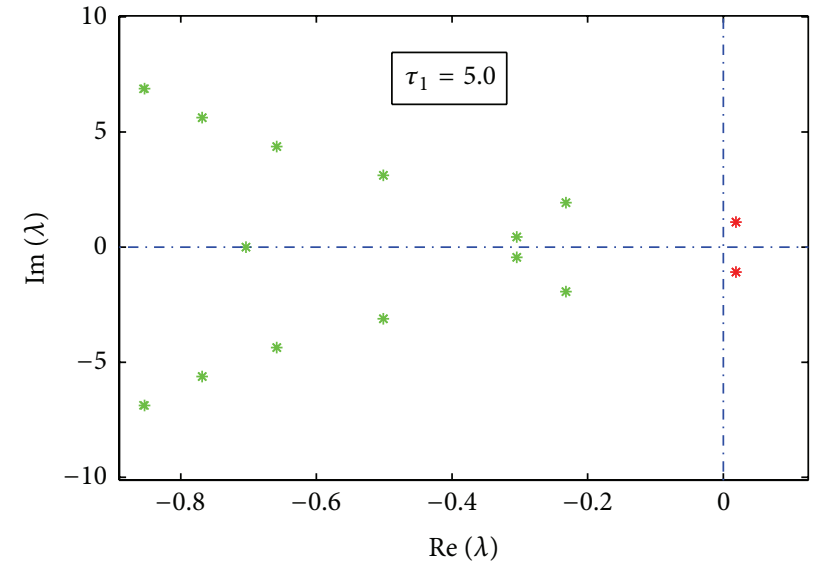

(b)

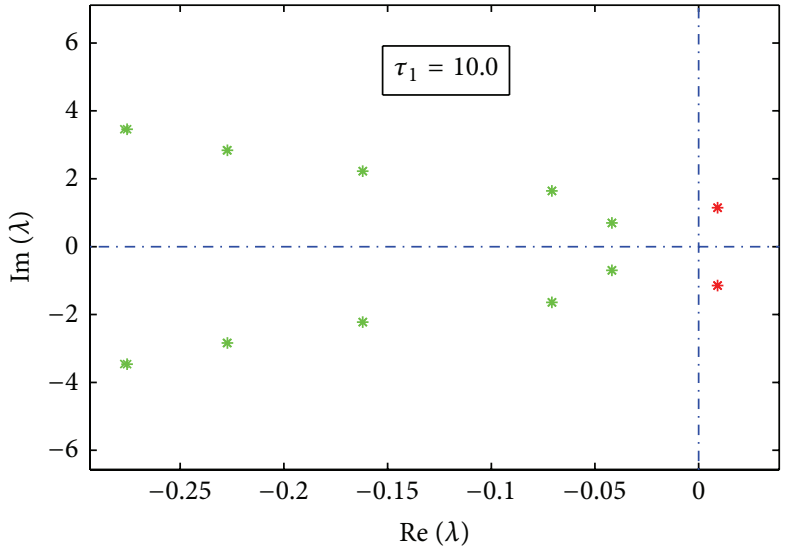

(d)

FIGURE 8: Distribution of partial eigenvalues for the fixed coupling weight $c=0.8$ and time delay $\tau_{2}=0$, where the asterisk in green represents the eigenvalues with negative real part and one with positive real part is in red color. The other parameters are fixed as $a=0.33, b=1$, and $\gamma=0.47$.

Figure 8(c) shows the eigenvalues are $-0.0133 \pm 0.8189 i$ for the fixed delay $\tau_{1}=8.0$. The neural system obtains again the resting state. Further, if delay $\tau_{1}$ is fixed as $\tau_{1}=10.0$, the maximum eigenvalues are the conjugate pair with positive part $9.0902 \times 10^{-3} \pm 1.1452 i$, as shown in Figure $8(\mathrm{~d})$. The system (1) exhibits the periodic spiking. In a word, the increase of time delay can induce the stability switches between resting state and periodic activity on the neural system (1).

Furthermore, we fix the time delay $\tau_{1}=\tau_{1}^{*}=0.5$ and find the combined effects of delay $\tau_{2}$ and coupling weight $c$ on the dynamic behavior of neural system (1). The figures of function $G$ and eigenvalues real parts are displayed in Figure 9 for the different coupling weights $c$. Figure 9 (a) shows that the curve $G$ with $c=0.2$. There is no intersection point when $\omega>0$ holds. It implies that the function $G$ has no positive root. The real parts of all eigenvalues have the negative part, as shown in Figure 9(b). The equilibrium point of system (1) is locally asymptotically stable for the arbitrary delay $\tau_{2}$. It is called the delay $\tau_{2}$-independent stability. With the coupling weight $c$ increasing to 0.8 , the curve determined by function
$G$ has two positive roots shown in Figure 9(c). It follows from Figure $9(\mathrm{~d})$ that there exist the delay $\tau_{2}$ intervals in which the eigenvalues have the negative parts. The trivial equilibrium point switches from stable to unstable, and then back to stable state with delay $\tau_{2}$ increasing. The neural system exhibits the stability switches between resting state and periodic spiking. When coupling weight $c$ is increased to 1.8 , function $G$ has two pairs of positive roots, as shown in Figure 9(e). However, at this time, the maximum eigenvalues of the characteristic equation (13) for the fixed delay $\tau_{2}=0$ have the positive real part $\operatorname{Re}(\lambda)=0.2787$. The equilibrium point of system (1) is instable for any delay $\tau_{2}$, as shown in Figure 9(f).

Additionally, it follows from Figure 10 that the time delay has the different effect on the periodic activity in the FHN neural system (1). When the coupling weight and time delay are chosen as $c=1.0, \tau_{1}=1.0$, the eigenvalues of the maximum real parts are changed from negative value to positive ones, as shown in Figure 10(a). It implies that the system behavior will evolve from the resting state into periodic spiking. That is to say, time delay promotes the occurrence of the periodic activity in the delay-coupling FHN 


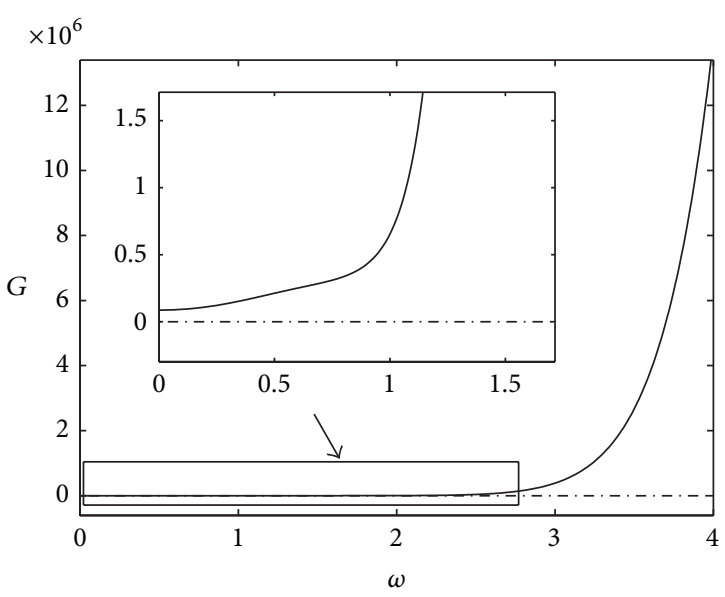

(a)

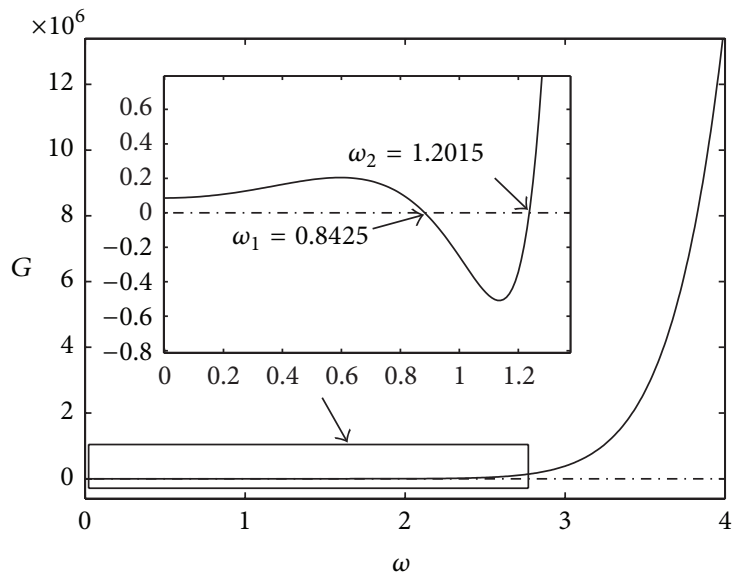

(c)

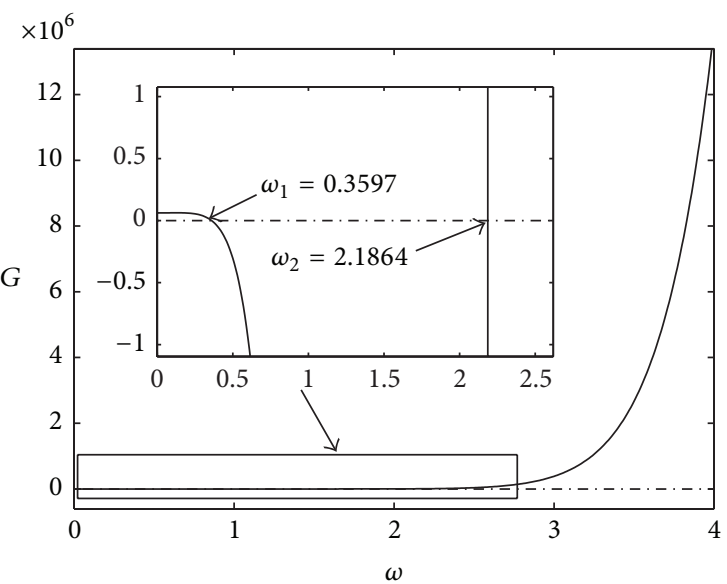

(e)

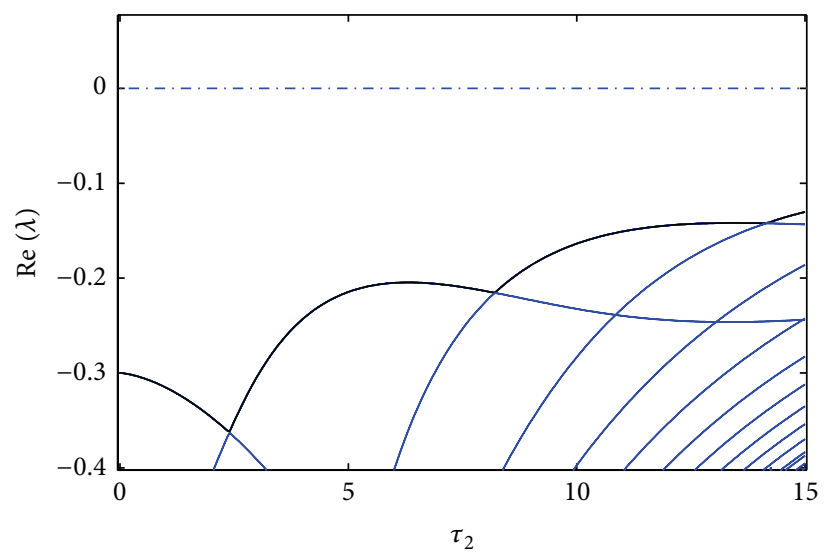

(b)

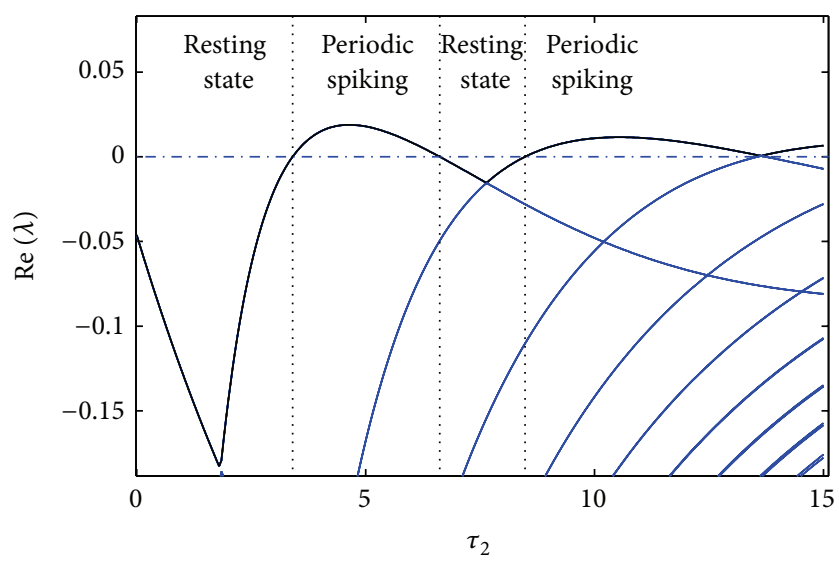

(d)

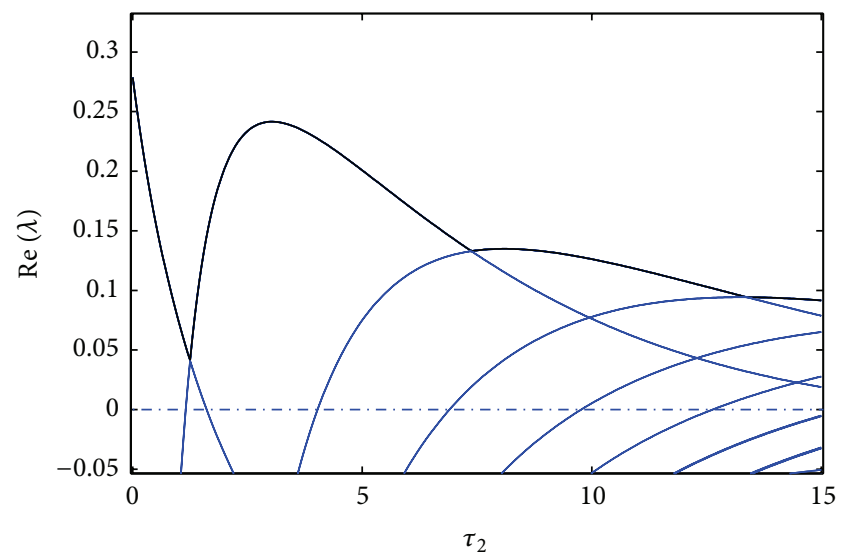

(f)

Figure 9: Function $G$ curve ((a), (c), and (e)) and eigenvalues real parts with $\tau_{2}$ varying ((b), (d), and (f)) for the different coupling weights (a)-(b) $c=0.2$, (c)-(d) $c=0.8$, and (e)-(f) $c=1.8$. The other parameters are fixed as $a=0.33, b=1, \gamma=0.47$, and $\tau_{1}=0.5$.

neural system. However, for the parameter values of $c=1.2$ and $\tau_{1}=0.1$, the system has the periodic activity with the nondelay $\tau_{2}=0$. Increasing delay $\tau_{2}$, the eigenvalue of the maximum real parts exhibits the negative value, as shown in
Figure 10(b). It implies that the trivial equilibrium point obtains the stability by the increasing delay $\tau_{2}$. Time delay suppresses the system dynamic from periodic activity into resting state, which can be used to control the neural system. 


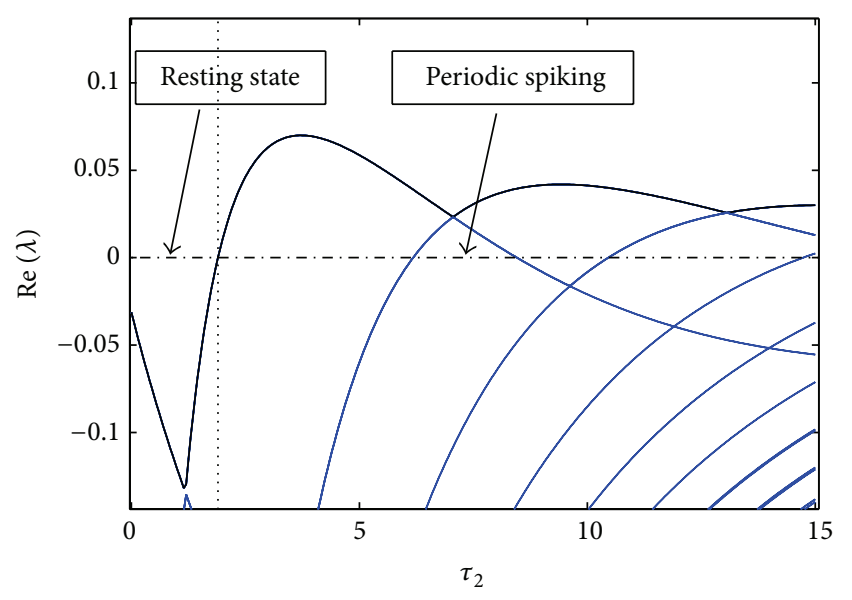

(a)

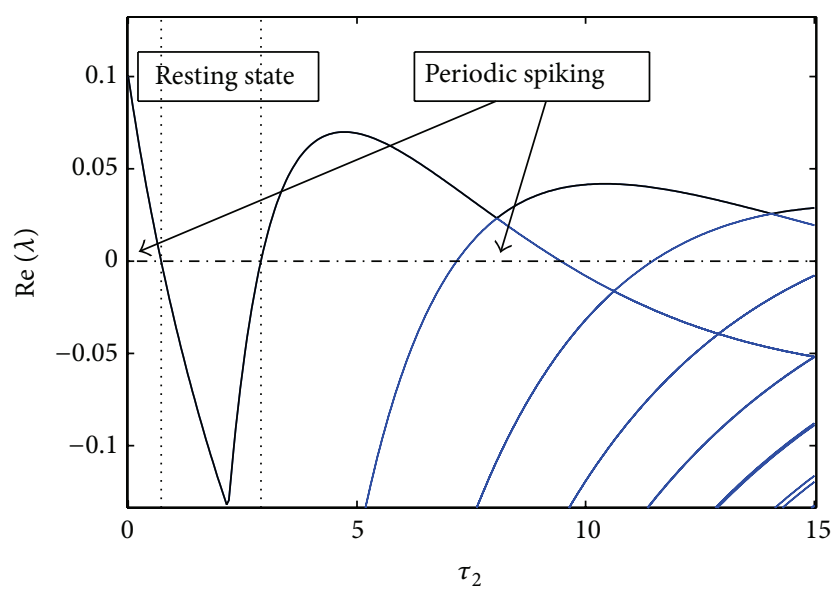

(b)

FIGURE 10: Eigenvalues of the maximum real parts show that the delay $\tau_{2}$ increasing promotes the system dynamic from resting state into periodic spiking for $c=1.0, \tau_{1}=1.0$ (a) and suppresses ones from periodic activity into resting state for $c=1.2, \tau_{1}=0.1(\mathrm{~b})$, where the other parameters are fixed as $a=0.33, b=1$, and $\gamma=0.47$.

\section{Conclusion}

Time delay is an inevitable factor in the signal transmission between neurons. The neural system with time delay exhibits the rich dynamical behaviors. In this paper, a coupled FHN neural system with two delays has been proposed. The analyses of the number of equilibrium points illustrate that the neural system has a unique equilibrium and three equilibria for the different values of coupling weights. It exhibits the multiple equilibrium points employing the saddle-node bifurcation of nontrivial equilibrium point and the transcritical bifurcation of the trivial point. Further, the stability of equilibrium point is analyzed employing the corresponding characteristic equation. Some stability criteria involving the multiple delays and coupling weight are obtained. The results show that the FHN neural system exhibits the parameter regions involved the delay-independence stability and delaydependence stability. Time delay increasing can induce the stability switches between resting state and periodic activity.
Finally, numerical simulations are taken to support the theoretical results.

\section{Conflict of Interests}

The authors declare that there is no conflict of interests regarding the publication of this paper.

\section{Acknowledgments}

The authors would like to thank the editor and the reviewers for their valuable suggestions and comments.

\section{References}

[1] R. FitzHugh, "Impulses and physiological states in theoretical models of nerve membrane," Biophysical Journal, vol. 1, no. 6, pp. 445-466, 1961.

[2] J. Nagumo, S. Arimoto, and S. Yoshizawa, "An active pulse transmission line simulating nerve axon," Proceedings of the IRE, vol. 50, no. 10, pp. 2061-2070, 1962.

[3] A. L. Hodgkin and A. F. Huxley, "A quantitative description of membrane current and its application to conduction and excitation in nerve," The Journal of Physiology, vol. 117, no. 4, pp. 500-544, 1952.

[4] A. N. Bautin, "Qualitative investigation of a particular nonlinear system," Journal of Applied Mathematics and Mechanics, vol. 39, no. 4, pp. 606-615, 1975.

[5] J. Duarte, L. Silva, and J. S. Ramos, "Types of bifurcations of FitzHugh-Nagumo maps," Nonlinear Dynamics, vol. 44, no. 14, pp. 231-242, 2006.

[6] T. Ueta, H. Miyazaki, T. Kousaka, and H. Kawakami, "Bifurcation and chaos in coupled BVP oscillators," International Journal of Bifurcation and Chaos in Applied Sciences and Engineering, vol. 14, no. 4, pp. 1305-1324, 2004.

[7] T. Ueta and H. Kawakami, "Bifurcation in asymmetrically coupled BVP oscillators," International Journal of Bifurcation and Chaos in Applied Sciences and Engineering, vol. 13, no. 5, pp. 1319-1327, 2003.

[8] S. Tsuji, T. Ueta, and H. Kawakami, "Bifurcation analysis of current coupled BVP oscillators," International Journal of Bifurcation and Chaos in Applied Sciences and Engineering, vol. 17, no. 3, pp. 837-850, 2007.

[9] D. Yang, "Self-synchronization of coupled chaotic FitzHughNagumo systems with unreliable communication links," Communications in Nonlinear Science and Numerical Simulation, vol. 18, no. 10, pp. 2783-2789, 2013.

[10] C. Xu and P. Li, "Dynamics in a delayed neural network model of two neurons with inertial coupling," Abstract and Applied Analysis, vol. 2012, Article ID 689319, 17 pages, 2012.

[11] J. Liang, Z. Chen, and Q. Song, "State estimation for neural networks with leakage delay and time-varying delays," Abstract and Applied Analysis, vol. 2013, Article ID 289526, 9 pages, 2013.

[12] N. Burić and D. Todorović, "Dynamics of FitzHugh-Nagumo excitable systems with delayed coupling," Physical Review E, vol. 67, Article ID 066222, 2003.

[13] N. Buric and D. Todorovic, "Bifurcations due to small timelag in coupled excitable systems," International Journal of Bifurcation and Chaos, vol. 15, no. 5, pp. 1775-1785, 2005. 
[14] N. Burić, I. Grozdanović, and N. Vasović, “Type I vs. type II excitable systems with delayed coupling," Chaos, Solitons \& Fractals, vol. 23, no. 4, pp. 1221-1233, 2005.

[15] Q. Wang, Q. Lu, G. Chen, Z. feng, and L. Duan, "Bifurcation and synchronization of synaptically coupled FHN models with time delay," Chaos, Solitons and Fractals, vol. 39, no. 2, pp. 918-925, 2009.

[16] D. Fan and L. Hong, "Hopf bifurcation analysis in a synaptically coupled FHN neuron model with delays," Communications in Nonlinear Science and Numerical Simulation, vol. 15, no. 7, pp. 1873-1886, 2010.

[17] B. Zhen and J. Xu, "Simple zero singularity analysis in a coupled FitzHugh-Nagumo neural system with delay," Neurocomputing, vol. 73, no. 4-6, pp. 874-882, 2010.

[18] B. Zhen and J. Xu, "Bautin bifurcation analysis for synchronous solution of a coupled FHN neural system with delay," Communications in Nonlinear Science and Numerical Simulation, vol. 15, no. 2, pp. 442-458, 2010.

[19] Y. Lin, "Periodic oscillation analysis for a coupled FHN network model with delays," Abstract and Applied Analysis, vol. 2013, Article ID 276972, 6 pages, 2013.

[20] Y. Li and W. Jiang, "Hopf and Bogdanov-Takens bifurcations in a coupled FitzHugh-Nagumo neural system with delay," Nonlinear Dynamics, vol. 65, no. 1-2, pp. 161-173, 2011.

[21] C. D. Yang, J. Qiu, and J. W. Wang, "Robust $H_{\infty}$ control for a class of nonlinear distributed parameter systems via proportional-spatial derivative control approach," Abstract and Applied Analysis, vol. 2014, Article ID 631071, 8 pages, 2014.

[22] Z. G. Song and J. Xu, "Codimension-two bursting analysis in the delayed neural system with external stimulations," Nonlinear Dynamics, vol. 67, no. 1, pp. 309-328, 2012.

[23] Z. G. Song and J. Xu, "Bursting near bautin bifurcation in a neural network with delay coupling," International Journal of Neural Systems, vol. 19, no. 5, pp. 359-373, 2009.

[24] Z.-G. Song and J. Xu, "Stability switches and multistability coexistence in a delay-coupled neural oscillators system," Journal of Theoretical Biology, vol. 313, no. 21, pp. 98-114, 2012.

[25] Z. Song and J. Xu, "Stability switches and double Hopf bifurcation in a two-neural network system with multiple delays," Cognitive Neurodynamics, vol. 7, no. 6, pp. 505-521, 2013. 


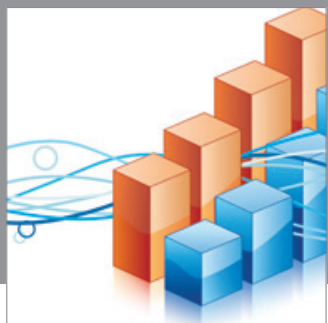

Advances in

Operations Research

mansans

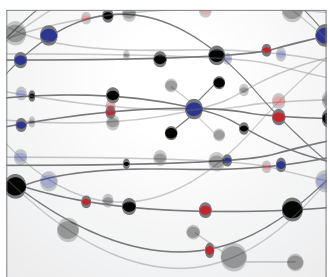

The Scientific World Journal
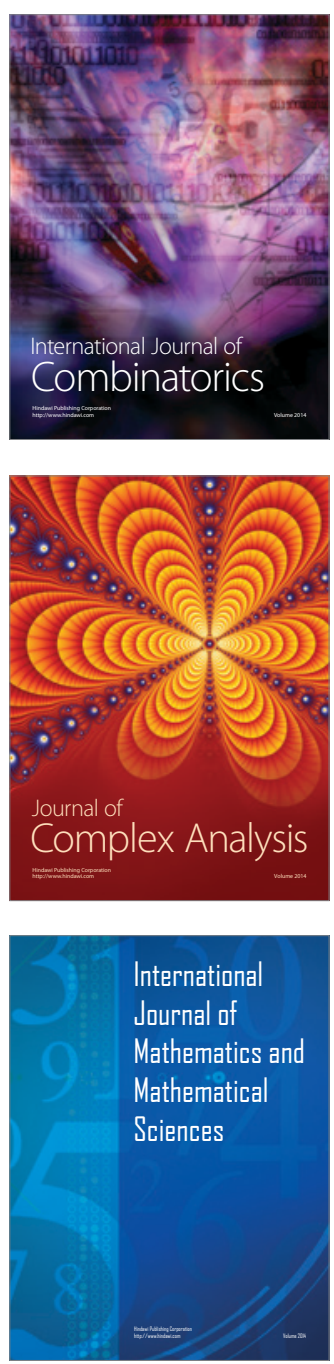
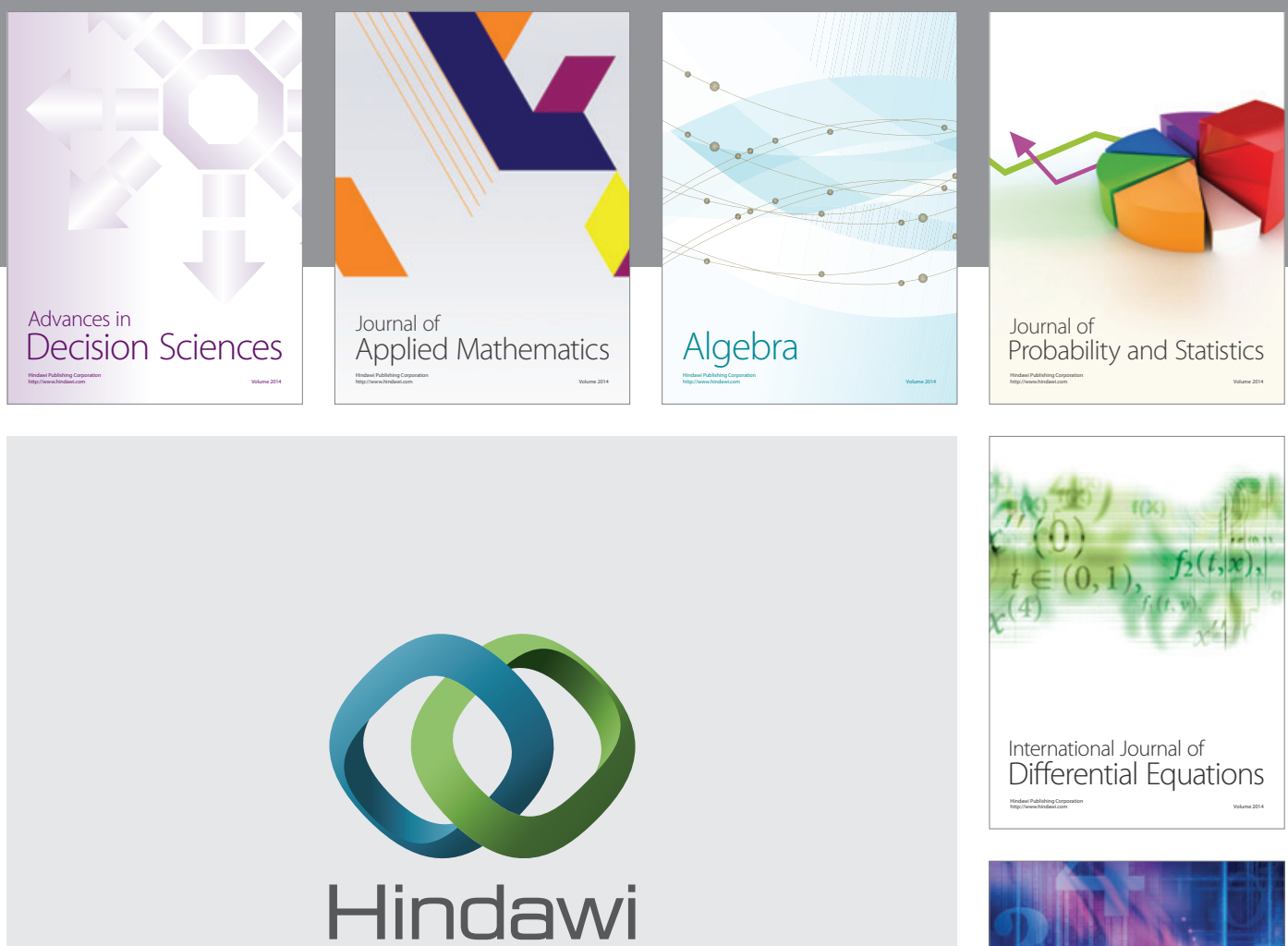

Submit your manuscripts at http://www.hindawi.com
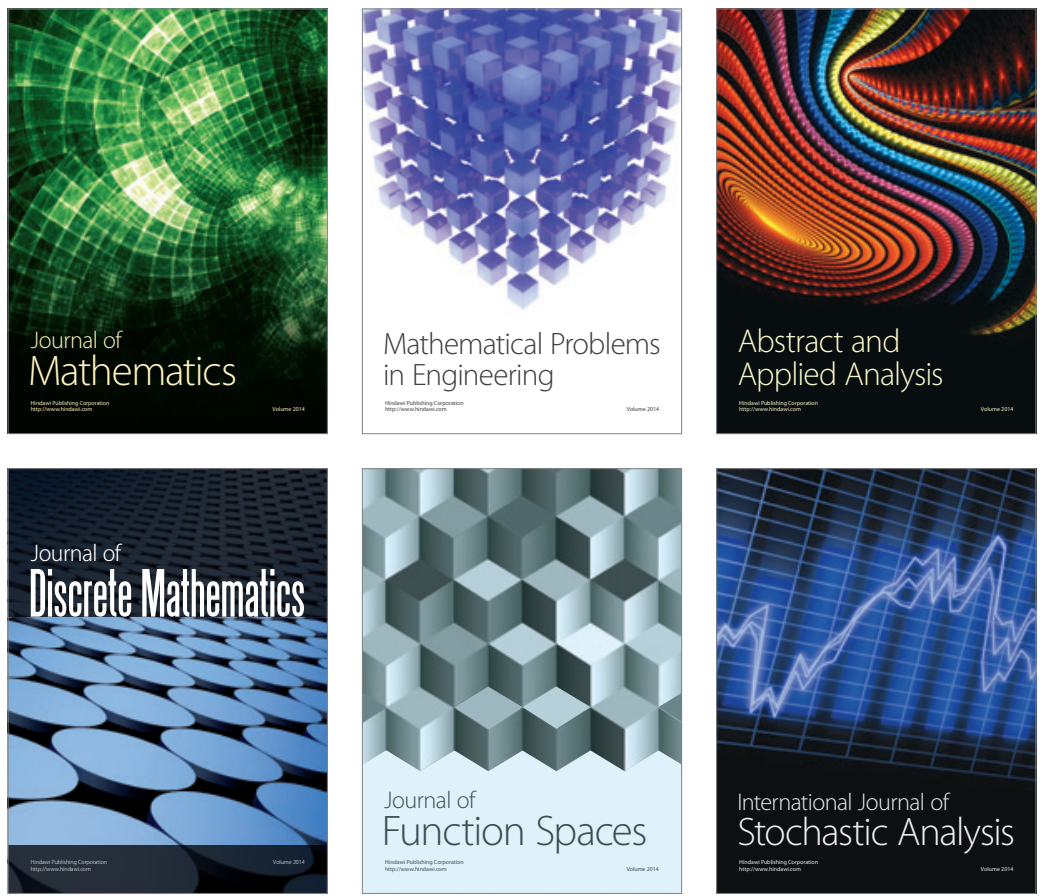

Journal of

Function Spaces

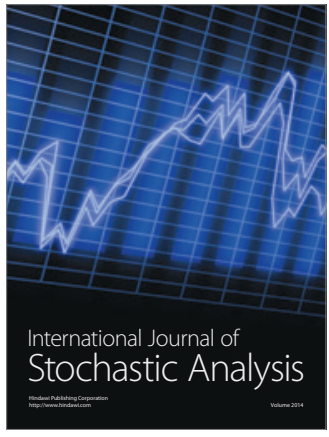

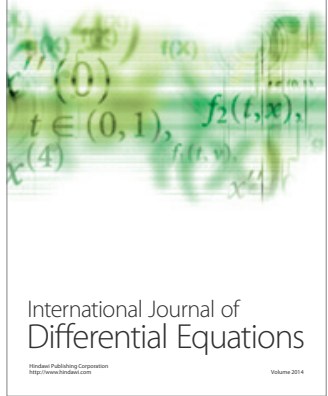
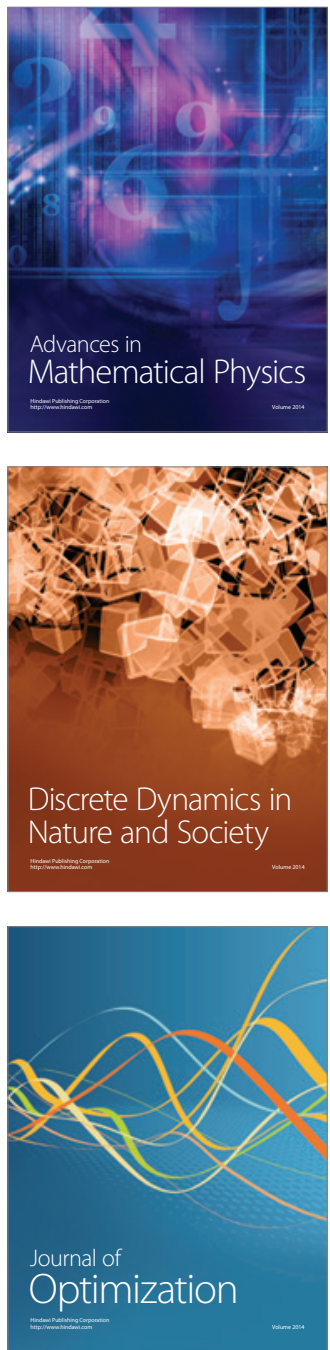